\title{
AN UNFITTED FORMULATION FOR THE INTERACTION OF AN INCOMPRESSIBLE FLUID WITH A THICK STRUCTURE VIA AN XFEM/DG APPROACH*
}

\author{
STEFANO ZONCA ${ }^{\dagger}$, CHRISTIAN VERGARA $^{\dagger}$, AND LUCA FORMAGGIA ${ }^{\dagger}$
}

\begin{abstract}
A numerical procedure that combines an extended finite element formulation and a discontinuous Galerkin technique is presented, with the final aim of providing an effective tool for the simulation of three-dimensional (3D) fluid-structure interaction problems. In this work we consider a thick structure immersed in a fluid. We describe the numerical models and discuss the specific implementation issues arising in three dimensions. Finally, 3D numerical results are provided to show the effectiveness of the approach.
\end{abstract}

Key words. unfitted meshes, extended finite element method, discontinuous Galerkin, fluidstructure interaction

AMS subject classifications. $65 \mathrm{M} 60,74 \mathrm{~F} 10,65 \mathrm{M} 85$

DOI. $10.1137 / 16 \mathrm{M} 1097602$

1. Introduction. The study of the mechanics of the heart valves and their interaction with blood is very important for understanding their functional behavior, for developing prosthetic valves, and for post-surgery feedback; see, e.g., [45, 28]. In this context, the leaflets of the aortic valve, which is at the interface between the left ventricle and the aorta, play a key role since they have a major influence on the blood fluid-dynamics in the proximity of the valve and along the aortic arch $[29,40,46,15]$. The numerical simulation of such a scenario could provide important quantitative information about the fluid-structure interaction (FSI) arising between blood and leaflets. Unlike the vascular case, here the FSI problem requires dealing with some issues, namely, the large displacements involving the leaflets, their small thickness, and the treatment of the contact.

A classical approach to deal with the vascular FSI problem relies on the generation of a single mesh with a fitted interface between fluid and vessel wall. While for the structure problem a Lagrangian formulation is usually considered, for the fluid one an arbitrary Lagrangian Eulerian (ALE) approach is employed to take care of the movement of the interface and the resulting deformation of the fluid mesh induced by the displacement of the structure [17]. However, in the valve FSI problem this strategy may lead to a very distorted fluid mesh and in some cases a remeshing procedure may be necessary [27]. A different approach consists in considering unfitted meshes, where the fluid mesh is fixed on the background while the structure one is free to move independently. Within these methods, the immersed boundary (IB) $[34,35,5,31,6,23,25,4]$ and the fictitious domain (FD) methods [25, 22] are two effective techniques that were successfully employed in the case of thin valve leaflets;

* Submitted to the journal's Computational Methods in Science and Engineering section October 16, 2016; accepted for publication (in revised form) September 15, 2017; published electronically January $11,2018$.

http://www.siam.org/journals/sisc/40-1/M109760.html

Funding: That authors gratefully acknowledge the financial support of the Italian MIUR by the grant PRIN12, number 20121289A4XL, "Mathematical and numerical models of the cardiovascular system, and their clinical applications." CV has been also partially supported by INDAM-GNCS.

${ }^{\dagger}$ MOX - Dipartimento di Matematica, Politecnico di Milano, Piazza L. da Vinci 32, 20133 Milano, Italy (stefano.zonca@polimi.it, luca.formaggia@polimi.it, christian.vergara@polimi.it). 
see, e.g., [43, 14, 36, 3, 8, 24]. A more recent methodology is based on an unfitted formulation that allows one to treat nonconforming, overlapping/unfitted meshes by writing the weak formulation of fluid and structure problems in their physical domains. This possibly leads to mesh elements with complex shape, allowing one to maintain the accuracy of the standard finite element method; see, e.g., [1, 11, 30].

The employment of an ALE approach allows one to easily treat the geometrical coupling at the fluid-structure interface as well as the imposition of the physical coupling conditions (no-slip condition and third Newton law). However, it is not suited to handle large displacements and the contact among structures. On the other hand, the use of an unfitted approach avoids the issues of moving or remeshing the fluid mesh, allowing one to manage in an easier way the movement, and possibly the contact, of the structures. In this case, the coupling at the interface is more delicate, both on the geometrical and numerical points of view: some approaches, such as FD, result in a loss of accuracy; other approaches, such as the extended finite element method (XFEM) $[26,1]$ or IB, reconstruct exactly the position of the interface with respect to the fluid mesh allowing a more accurate solution, but are more complex to implement.

In this work, we consider the case of a three-dimensional (3D) structure immersed in a fluid where the solid mesh overlaps the fluid one and the interface is fitted only to the solid mesh. Due to the small thickness of the 3D structure, which may be smaller than the characteristic fluid mesh size, the solid may split a fluid element into two subparts, thus generating two (or more) fluid polyhedra with the solid in between. (In what follows we refer to this kind of elements as split tetrahedra.) To manage this situation, we propose to use XFEM, where the degrees of freedom (dofs) of the split elements are duplicated, allowing one to represent a discontinuity within the element accurately. We notice that in the literature, other, more general, definitions of XFEM have been provided [21]. Here we will refer to XFEM only when the duplication of the dofs is considered.

To glue the solution at the physical interface, we employ a discontinuous Galerkin (DG) technique. The use of an unfitted formulation in combination with the XFEM and the DG techniques has been reported in [1] for the case of a membrane structure. In [37], the authors employ a similar formulation in 3D for the incompressible NavierStokes equations solely. To the best of our knowledge, this strategy is here employed for the first time for a FSI problem with thick structure.

The paper is organized as follows. In section 2 we present the fluid-structure interaction problem and the corresponding XFEM/DG discretization. In section 3 we describe how to solve the major technical issues encountered by the proposed method. In section 4 we show several 3D numerical tests to assess and validate the proposed method. In particular, we consider both a geometrically fixed and a moving structure. In this first work, we consider only moderate Reynolds numbers $(\simeq 10)$. Finally, section 5 is devoted to conclusions and limitations.

2. Numerical formulation. In section 2.1 , we present the numerical formulation of a time-dependent fluid-structure interaction problem with thick structure in the case of geometrically fixed immersed structure. The case of completely moving structure is treated in section 2.2. We consider the incompressible Navier-Stokes equations for the fluid and the linear elastic model for the structure.

\subsection{The case of geometrically fixed structure.}

2.1.1. Governing equations. Referring to Figure 1 , we consider a fluid domain $\Omega^{f}$ and a structure domain $\Omega^{s}$ such that $\Omega=\Omega^{f} \cup \Omega^{s} \subset \mathbb{R}^{d}, d=2,3$, and $\Sigma=\bar{\Omega}^{f} \cap \bar{\Omega}^{s}$ 


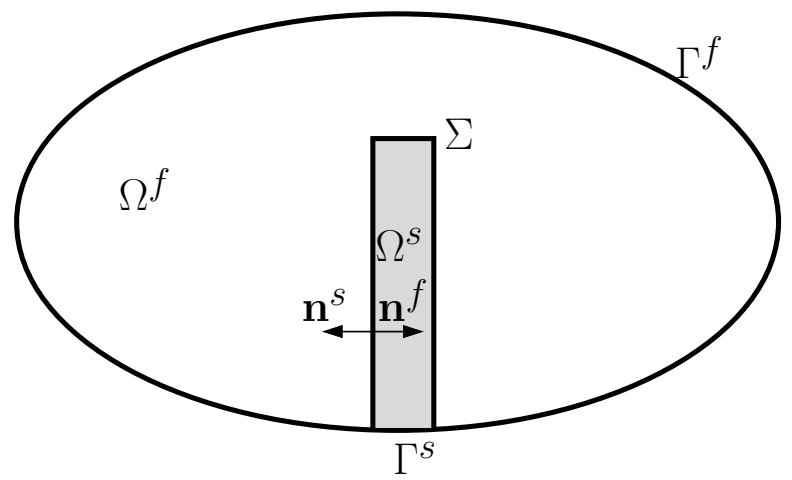

FIG. 1. Sketch of the fluid and structure domain $\Omega^{f}$ and $\Omega^{s}$ with the fluid-structure interface $\Sigma$.

is the fluid-structure interface. We denote by $\partial \Omega^{f}$ and $\partial \Omega^{s}$ the boundary of the fluid and solid domain, respectively, and we define $\Gamma^{f}=\partial \Omega^{f} \backslash \Sigma$ and $\Gamma^{s}=\partial \Omega^{s} \backslash \Sigma$. Finally, we indicate with $\mathbf{n}^{f}$ and $\mathbf{n}^{s}$ the outward unit normal to the domain $\Omega^{f}$ and $\Omega^{s}$, respectively. On the interface $\Sigma$ we have $\mathbf{n}^{f}=-\mathbf{n}^{s}=\mathbf{n}$. We notice that all these quantities are fixed and do not change in time.

The fluid-structure interaction problem reads as follows: find the fluid velocity $\mathbf{u}: \Omega^{f} \times(0, T] \rightarrow \mathbb{R}^{d}$, the fluid pressure $p: \Omega^{f} \times(0, T] \rightarrow \mathbb{R}$, and the solid displacement $\mathbf{d}: \Omega^{s} \times(0, T] \rightarrow \mathbb{R}^{d}$, such that

$$
\begin{array}{lc}
\rho^{f} \partial_{t} \mathbf{u}+\rho^{f} \mathbf{u} \cdot \nabla \mathbf{u}-\nabla \cdot \mathbf{T}^{f}(\mathbf{u}, p)=\mathbf{0} & \text { in } \Omega^{f} \times(0, T], \\
\nabla \cdot \mathbf{u}=0 & \text { in } \Omega^{f} \times(0, T], \\
\mathbf{u}=\mathbf{0} & \text { on } \Gamma^{f} \times(0, T] ; \\
\rho^{s} \partial_{t t} \mathbf{d}-\nabla \cdot \mathbf{T}^{s}(\mathbf{d})=\mathbf{0} & \text { in } \Omega^{s} \times(0, T], \\
\mathbf{d}=\mathbf{0} & \text { on } \Gamma^{s} \times(0, T] ; \\
\mathbf{u}=\dot{\mathbf{d}} & \text { on } \Sigma \times(0, T], \\
\mathbf{T}^{f}(\mathbf{u}, p) \mathbf{n}^{f}=-\mathbf{T}^{s}(\mathbf{d}) \mathbf{n}^{s} & \text { on } \Sigma \times(0, T],
\end{array}
$$

where (1a)-(1c) are the Navier-Stokes equations, (1d)-(1e) the equations of elastodynamics, and (1f) $-(1 \mathrm{~g})$ the physical coupling conditions. Moreover, we have $T>0$, $\rho^{f}$ and $\rho^{s}$ are the fluid and structure densities, $\mathbf{T}^{f}(\mathbf{u}, p)=-p \mathbf{I}+2 \mu^{f} \mathbf{D}(\mathbf{u})$ is the fluid Cauchy stress tensor, $\mathbf{T}^{s}(\mathbf{d})=\lambda^{s}(\nabla \cdot \mathbf{d}) \mathbf{I}+2 \mu^{s} \mathbf{D}(\mathbf{d})$ is the solid stress tensor, $\mathbf{D}(\mathbf{w})=\frac{1}{2}\left(\nabla \mathbf{w}+\nabla \mathbf{w}^{T}\right), \mu^{f}$ is the fluid dynamic viscosity, $\lambda^{s}, \mu^{s}>0$ are the Lamé parameters, $\dot{\mathbf{d}}=\partial_{t} \mathbf{d}$, and for the sake of simplicity we have considered homogeneous Dirichlet conditions on $\Gamma^{f}$ and $\Gamma^{s}$.

The problem is completed with the initial conditions $\mathbf{u}(\mathbf{x}, 0)=\mathbf{u}^{0}(\mathbf{x}), \mathbf{d}(\mathbf{x}, 0)=$ $\mathbf{d}^{0}(\mathbf{x})$ and $\dot{\mathbf{d}}(\mathbf{x}, 0)=\mathbf{v}^{0}(\mathbf{x})$.

2.1.2. Spatial discretization. We consider the spaces $\mathbf{V}=\left[H_{\Gamma^{f}}^{1}\left(\Omega^{f}\right)\right]^{d}, Q=$ $L^{2}\left(\Omega^{f}\right)$, and $\mathbf{W}=\left[H_{\Gamma^{s}}^{1}\left(\Omega^{s}\right)\right]^{d}$, where $H_{\Gamma^{f}}^{1}\left(\Omega^{f}\right)=\left\{v \in H^{1}\left(\Omega^{f}\right), v_{\mid \Gamma^{f}}=0\right\}$, and $H_{\Gamma^{s}}^{1}\left(\Omega^{s}\right)=\left\{v \in H^{1}\left(\Omega^{s}\right), v_{\mid \Gamma^{s}}=0\right\}$. The weak formulation of the problem given by (1) reads as follows: for $t \in(0, T]$, find $(\mathbf{u}(t), p(t), \mathbf{d}(t)) \in \mathbf{V} \times Q \times \mathbf{W}$ such that $\mathbf{u}=\dot{\mathbf{d}}$ 


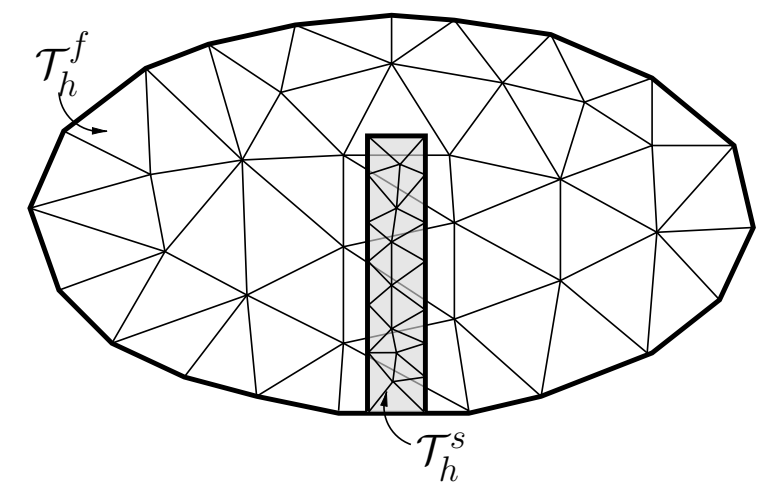

FIG. 2. The structure mesh $\mathcal{T}_{h}^{s}$ overlaps the fluid mesh $\mathcal{T}_{h}^{f}$.

on $\Sigma$, and

$$
\left\{\begin{array}{l}
\rho^{f}\left(\partial_{t} \mathbf{u}, \mathbf{v}\right)_{\Omega^{f}}+a^{f}(\mathbf{u}, \mathbf{v})+b(p, \mathbf{v})-b(q, \mathbf{u})+c(\mathbf{u}, \mathbf{u}, \mathbf{v}) \\
+\rho^{s}\left(\partial_{t t} \mathbf{d}, \mathbf{w}\right)_{\Omega^{s}}+a^{s}(\mathbf{d}, \mathbf{w})=0
\end{array}\right.
$$

$\forall(\mathbf{v}, q, \mathbf{w}) \in \mathbf{V} \times Q \times \mathbf{W}$ such that $\mathbf{v}_{\mid \Sigma}=\mathbf{w}_{\mid \Sigma}$. Here, with $\cdot \mid \Sigma$ we indicate the trace on $\Sigma$ and we have indicated by $(\cdot, \cdot)_{\Omega^{i}}, i=f, s$, the $L^{2}$ product over $\Omega^{i}$. Moreover, we have introduced the bilinear forms $a^{f}: \mathbf{V} \times \mathbf{V} \rightarrow \mathbb{R}, b: Q \times \mathbf{V} \rightarrow \mathbb{R}$ and $a^{s}: \mathbf{W} \times \mathbf{W} \rightarrow \mathbb{R}$ defined as

$$
\begin{aligned}
& a^{f}(\mathbf{u}, \mathbf{v})=2 \mu^{f}(\mathbf{D}(\mathbf{u}), \mathbf{D}(\mathbf{v}))_{\Omega^{f}} \\
& b(p, \mathbf{v})=-(p, \nabla \cdot \mathbf{v})_{\Omega^{f}} \\
& a^{s}(\mathbf{d}, \mathbf{w})=\lambda^{s}(\nabla \cdot \mathbf{d}, \nabla \cdot \mathbf{w})_{\Omega^{s}}+2 \mu^{s}(\mathbf{D}(\mathbf{d}), \mathbf{D}(\mathbf{w}))_{\Omega^{s}}
\end{aligned}
$$

and the trilinear form $c: \mathbf{V} \times \mathbf{V} \times \mathbf{V} \rightarrow \mathbb{R}$ defined as

$$
c(\mathbf{z}, \mathbf{u}, \mathbf{v})=\rho^{f}(\mathbf{z} \cdot \nabla \mathbf{u}, \mathbf{v})_{\Omega_{f}} .
$$

For further details on the weak formulation and its analysis, see, e.g., [19].

To ease the presentation, we assume that $\Omega^{f}, \Omega^{s}$, and $\Sigma$ are polyhedral. We denote by $\mathcal{T}_{h}^{s}$ the solid mesh that covers the domain $\Omega^{s}$ and is fitted to $\partial \Omega^{s}$, and by $\mathcal{T}_{h}^{f}$ the fluid mesh that covers the whole domain $\Omega$ and is fitted to $\Gamma^{f}$, but in general not to $\Sigma$ and $\Gamma^{s}$. We indicate with $h>0$ the space discretization step which is a function that may vary among the elements $K$ of the meshes and between the fluid and structure meshes. As result, the solid mesh $\mathcal{T}_{h}^{s}$ overlaps the fluid mesh $\mathcal{T}_{h}^{f}$; see Figure 2.

We also introduce the following background fluid mesh,

$$
\mathcal{G}_{h}=\left\{K: K \in \mathcal{T}_{h}^{f}, K \cap \Sigma \neq \emptyset, K \cap \Omega^{f} \text { is a nonconnected set }\right\},
$$

that consists of all the elements $K$ in $\mathcal{T}_{h}^{f}$ cut by the interface $\Sigma$ which are split elements. This means that each fluid element $K \in \mathcal{G}_{h}$ is split into $N^{K} \geq 2$ fluid subparts, which in, are polyhedra; see Figure 3, where $N^{K}=2$. Let us denote by $P_{i}^{K}, i=1, \ldots, N^{K}$, the polyhedra of a fluid split element $K$; see Figure 3, right. We define by $\mathcal{G}_{h}^{P}$ the union of all such polyhedra $P_{i}^{K}$ for $i=1, \ldots, N^{K}$ and for each 



FIG. 3. Left: representation of the background mesh $\mathcal{G}_{h}$. Center: representation of the nonconnected mesh $\mathcal{G}_{h}^{P}$. Right: representation of a split element $K$ with two fluid subparts $P_{1}^{K}$ and $P_{2}^{K}$.
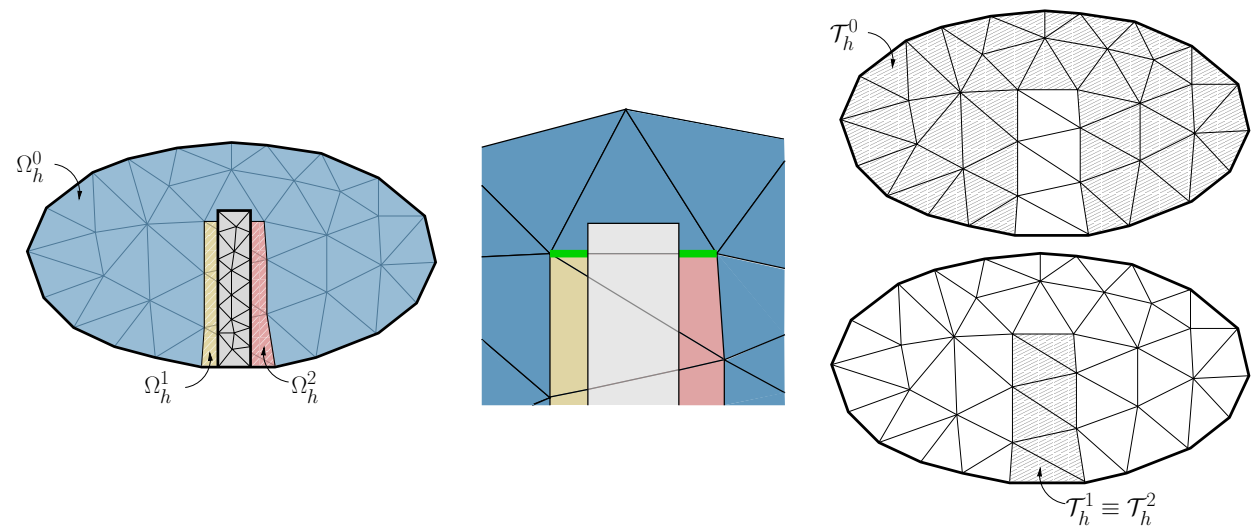

FIG. 4. Left: sketch of the sets $\Omega_{h}^{0}, \Omega_{h}^{1}$, and $\Omega_{h}^{2}$ in the case of $N^{f}=2$. Center: example of scenario where a continuous approximation is not possible for the fluid problem. Right: the shaded regions represent the meshes $\mathcal{T}_{h}^{0}$ (top) and $\mathcal{T}_{h}^{i}$ (bottom) in the case of $N^{f}=2$. In this case, $\mathcal{T}_{h}^{1}$ coincides with $\mathcal{T}_{h}^{2}$.

$K \in \mathcal{G}_{h}$. More precisely

$$
P \in \mathcal{G}_{h}^{P} \longleftrightarrow \exists K \in \mathcal{G}_{h} \text { s.t. } P \subset K \cap \Omega^{f} \text { is a connected set. }
$$

The set $\mathcal{G}_{h}^{P}$ in now partitioned into its $N^{f}=\max _{K} N^{K}$ connected subsets $\Omega_{h}^{i}$. For example, in Figure 4, left, we have $N^{f}=2$ connected subregions $\Omega_{h}^{1}$ and $\Omega_{h}^{2}$.

Moving from these definitions, we set

$$
\Omega_{h}^{0}=\Omega^{f} \backslash \bigcup_{K \in \mathcal{G}_{h}} K
$$

(see Figure 4, left), and we denote by $\mathcal{T}_{h}^{0}$ the smallest mesh composed of the elements $K \in \mathcal{T}_{h}^{f}$ that covers the set $\Omega_{h}^{0}$, i.e.,

$$
K \in \mathcal{T}_{h}^{0} \longleftrightarrow K \cap \bar{\Omega}_{h}^{0} \neq \emptyset
$$

see Figure 4, right. Finally, we denote by $\mathcal{T}_{h}^{i}$ for $i=1, \ldots, N^{f}$ the smallest mesh that consists of all the elements of $\mathcal{G}_{h}$ that covers the set $\Omega_{h}^{i}$, i.e.,

$$
K \in \mathcal{T}_{h}^{i} \longleftrightarrow K \cap \bar{\Omega}_{h}^{i} \neq \emptyset, \quad i=1, \ldots, N^{f} .
$$




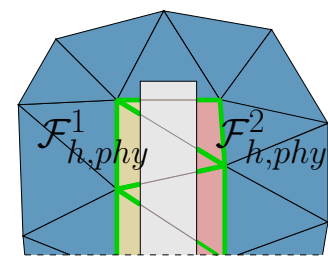

(a)

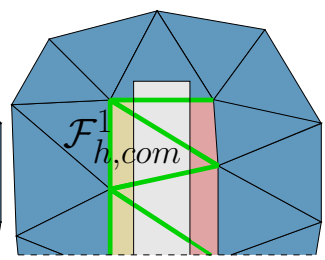

(b)

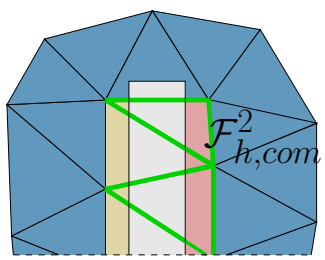

(c)

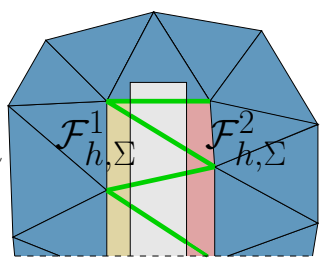

(d)

FIG. 5. Representation of the sets of faces involved in the integration (highlighted in green): (a) the physical faces $\mathcal{F}_{h, p h y}^{1}$ and $\mathcal{F}_{h, p h y}^{2}$; (b) the computational faces $\mathcal{F}_{h, \text { com }}^{1}$; (c) the computational faces $\mathcal{F}_{h, c o m}^{2}$; and $(d)$ the computational faces $\mathcal{F}_{h, \Sigma}^{1}$ and $\mathcal{F}_{h, \Sigma}^{2}$ intersected by $\Sigma$ which, in this example, coincide.

In this way, each element $K \in \mathcal{G}_{h}$ belongs to $N^{K}$ different meshes $\mathcal{T}_{h}^{i}$. We point out that $\Omega^{f}=\bigcup_{i=0, \ldots, N^{f}} \Omega_{h}^{i}$ and that $\Omega_{h}^{i} \cap \Omega_{h}^{j}=\emptyset \forall i \neq j$. We observe that the set covered by $\mathcal{T}_{h}^{i}$ is larger than the one covered by the corresponding $\Omega_{h}^{i}$; see Figure 4 , right. It is important to distinguish between the physical parts $\Omega_{h}^{i}$ and the computational ones $\mathcal{T}_{h}^{i}$, since some operators act on the former, while other operators, such as the stabilization terms, act on the latter. This requires one to be able to integrate over a portion of an element $K$ or a portion of a facet $F$. A detailed explanation of this point will be presented in section 3 . In what follows, we indicate with

- physical, the restriction of a geometrical entity of $\mathcal{T}_{h}^{i}$ on $\Omega_{h}^{i}$;

- computational, the entire geometrical entity in the mesh $\mathcal{T}_{h}^{i}$.

To ease the presentation, in what follows we suppose that $N^{f}=2$, so that we have only the sets $\Omega_{h}^{1}$ and $\Omega_{h}^{2}$; see Figure 4 , left.

Thanks to the above definitions, we can classify the faces in the region involved in the FSI coupling as follows:

- faces belonging to the fluid-structure interface $\Sigma$, where we impose weakly the continuity of the velocity and stresses by means of the DG formulation (see, e.g., $[10,11])$;

- $\mathcal{F}_{h, p h y}^{i}$, the physical part of the faces in $\mathcal{T}_{h}^{i}, i=1,2$, see the green edges in Figure 5(a), where we impose weakly the continuity of the fluid velocity and stresses by means of the DG formulation (see, e.g., $[2,16]$ );

- $\mathcal{F}_{h, \Sigma}^{i}$, the (computational) faces of $\mathcal{T}_{h}^{i}, i=1,2$, cut by the interface $\Sigma$, see the green edges in Figure 5(d), where the ghost penalty stabilization term is applied (see below);

- $\mathcal{F}_{h, c o m}^{i}$, the computational counterpart of the faces $\mathcal{F}_{h, p h y}^{i}, i=1,2$, see the green edges in Figure 5(b)-(c), where we apply the other stabilization terms (IP in our case; see below).

As we will explain in section 3.3, the dofs associated to the elements in $\mathcal{G}_{h}$ are duplicated according to the XFEM strategy: a set of dofs is used to compute the solution over $\mathcal{T}_{h}^{1}$, and a second set of dofs is used to compute the solution over $\mathcal{T}_{h}^{2}$. We observe that in some scenarios, like the one depicted in Figure 4, center, it is not possible to force continuity for the fluid problem across the edges represented in green. For example, using linear polynomial functions as done in this work (see below), on the upper part of such edges (element in blue) we have three dofs to represent the solution, whereas on the lower part (elements in yellow and pink) we have six dofs due to the XFEM doubling. For this reason, we apply a DG mortaring on these interfaces and, for simplicity, on all faces that belongs to $\mathcal{F}_{h, p h y}^{i}$ for $i=1,2$. 
We introduce the following spaces:

$$
X_{h}^{f}=\left\{v_{h} \in L^{2}\left(\Omega^{f}\right): v_{h} \in \mathcal{C}^{0}\left(\Omega_{h}^{0}\right), v_{h \mid K} \in \mathbb{P}_{1}(K), \forall K \in \mathcal{T}_{h}^{i} \text { for } i=0,1,2\right\}
$$

and

$$
X_{h}^{s}=\left\{v_{h} \in \mathcal{C}^{0}\left(\bar{\Omega}^{s}\right): v_{h \mid K} \in \mathbb{P}_{1}(K) \forall K \in \mathcal{T}_{h}^{s}\right\}
$$

For the approximation of the fluid velocity, fluid pressure, and solid displacement we consider the spaces

$$
\begin{gathered}
\mathbf{V}_{h}=\left\{\mathbf{v}_{h} \in\left[X_{h}^{f}\right]^{d}: \mathbf{v}_{h \mid \Gamma^{f}}=0\right\}, \quad Q_{h}=\left\{q_{h} \in X_{h}^{f}\right\}, \\
\mathbf{W}_{h}=\left\{\mathbf{w}_{h} \in\left[X_{h}^{s}\right]^{d}: \mathbf{w}_{h \mid \Gamma^{s}}=0\right\},
\end{gathered}
$$

respectively.

Further, we introduce some trace operators defined over an interface $\mathcal{I}$ that separates a domain $\Omega_{1,2}$ into $\Omega_{1}$ and $\Omega_{2}$, such that $\bar{\Omega}_{1,2}=\bar{\Omega}_{1} \cup \bar{\Omega}_{2}$ and $\bar{\Omega}_{1} \cap \bar{\Omega}_{2}=\mathcal{I}$. For a function $q$, we denote by $\llbracket \cdot \rrbracket_{\mathcal{I}}$ the jump and by $\{\{\cdot\}\}_{\mathcal{I}, \alpha}$ the $\alpha$-weighted mean across the interface $\mathcal{I}$, defined as

$$
\llbracket q \rrbracket_{\mathcal{I}}=q_{1}-q_{2}, \quad\{\{q\}\}_{\mathcal{I}, \alpha}=\alpha q_{1}+(1-\alpha) q_{2},
$$

where $q_{1}$ and $q_{2}$ are the traces of $q$ at the two sides of the interface and $\alpha \in[0,1]$. If the subscript $\alpha$ is not indicated, we assume that $\alpha=\frac{1}{2}$.

The space semidiscretization problem related to (1) reads as follows: for $t \in(0, T]$, find $\left(\mathbf{u}_{h}(t), p_{h}(t), \mathbf{d}_{h}(t)\right) \in \mathbf{V}_{h} \times Q_{h} \times \mathbf{W}_{h}$ such that

$$
\left\{\begin{array}{l}
\rho^{f}\left(\partial_{t} \mathbf{u}_{h}, \mathbf{v}_{h}\right)_{\Omega^{f}}+a^{f}\left(\mathbf{u}_{h}, \mathbf{v}_{h}\right)+b\left(p_{h}, \mathbf{v}_{h}\right)-b\left(q_{h}, \mathbf{u}_{h}\right)+c\left(\mathbf{u}_{h}, \mathbf{u}_{h}, \mathbf{v}_{h}\right) \\
+\rho^{s}\left(\partial_{t t} \mathbf{d}_{h}, \mathbf{w}_{h}\right)_{\Omega^{s}}+a^{s}\left(\mathbf{d}_{h}, \mathbf{w}_{h}\right) \\
\quad+c_{h}\left(\mathbf{u}_{h}, \mathbf{u}_{h}, \mathbf{v}_{h}\right)+\frac{\rho^{f}}{2}\left(\mathbf{u}_{h} \cdot \mathbf{n}, \dot{\mathbf{d}}_{h} \cdot \mathbf{v}_{h}\right)_{\Sigma}+s_{h}\left(\mathbf{u}_{h}, p_{h} ; \mathbf{v}_{h}, q_{h}\right)+g_{h}\left(\mathbf{u}_{h}, \mathbf{v}_{h}\right) \\
-\left(\alpha \mathbf{T}^{f}\left(\mathbf{u}_{h}, p_{h}\right) \mathbf{n}^{f}+(1-\alpha) \mathbf{T}^{s}\left(\mathbf{d}_{h}\right) \mathbf{n}^{f}, \mathbf{v}_{h}-\mathbf{w}_{h}\right)_{\Sigma} \\
-\left(\mathbf{u}_{h}-\dot{\mathbf{d}}_{h}, \alpha \mathbf{T}^{f}\left(\mathbf{v}_{h},-q_{h}\right) \mathbf{n}^{f}+(1-\alpha) \mathbf{T}^{s}\left(\mathbf{w}_{h}\right) \mathbf{n}^{f}\right)_{\Sigma} \\
+\frac{\gamma_{\Sigma} \mu^{f}}{h}\left(\mathbf{u}_{h}-\dot{\mathbf{d}}_{h}, \mathbf{v}_{h}-\mathbf{w}_{h}\right)_{\Sigma} \\
-\sum_{i=1,2} \sum_{F \in \mathcal{F}_{h, p h y}^{i}}\left(\left\{\left\{\mathbf{T}^{f}\left(\mathbf{u}_{h}, p_{h}\right)\right\}\right\}_{F} \mathbf{n}^{f}, \llbracket \mathbf{v}_{h} \rrbracket_{F}\right)_{F} \\
-\sum_{i=1,2} \sum_{F \in \mathcal{F}_{h, p h y}^{i}}\left(\llbracket \mathbf{u}_{h} \rrbracket_{F},\left\{\left\{\mathbf{T}^{f}\left(\mathbf{v}_{h},-q_{h}\right)\right\}\right\}_{F} \mathbf{n}^{f}\right)_{F} \\
+\sum_{i=1,2} \sum_{F \in \mathcal{F}_{h, p h y}^{i}} \frac{\gamma_{p h y}^{v} \mu^{f}}{h_{F}}\left(\llbracket \mathbf{u}_{h} \rrbracket_{F}, \llbracket \mathbf{v}_{h} \rrbracket_{F}\right)_{F}=0
\end{array}\right.
$$

$\forall\left(\mathbf{v}_{h}, q_{h}, \mathbf{w}_{h}\right) \in \mathbf{V}_{h} \times Q_{h} \times \mathbf{W}_{h}$. We have indicated by $\gamma_{\Sigma}>0$ and $\gamma_{p h y}^{v}>0$ the penalty parameters related to the interface $\Sigma$ and to the faces in $\mathcal{F}_{h, p h y}^{i}$, respectively. In (3), we have introduced the following:

- A correction of the convective term to maintain the condition $c(\mathbf{z}, \mathbf{v}, \mathbf{v})=$ $0 \forall \mathbf{v} \in \mathbf{V}, \mathbf{z} \in\{\mathbf{v} \in \mathbf{V}, \nabla \cdot \mathbf{v}=0\}$ in the discrete space (see $[42,16]$ ), defined 
as

$$
\begin{aligned}
c_{h}\left(\mathbf{z}_{h}, \mathbf{u}_{h}, \mathbf{v}_{h}\right)= & \frac{\rho^{f}}{2}\left(\left(\nabla \cdot \mathbf{z}_{h}\right) \mathbf{u}_{h}, \mathbf{v}_{h}\right)_{\Omega^{f}} \\
& -\sum_{i=1,2} \sum_{F \in \mathcal{F}_{h, p h y}^{i}} \rho^{f}\left(\left\{\left\{\mathbf{z}_{h}\right\}\right\}_{F} \cdot \mathbf{n} \llbracket \mathbf{u}_{h} \rrbracket_{F},\left\{\left\{\mathbf{v}_{h}\right\}\right\}_{F}\right)_{F} \\
& -\sum_{i=1,2} \sum_{F \in \mathcal{F}_{h, p h y}^{i}} \frac{\rho^{f}}{2}\left(\llbracket \mathbf{z}_{h} \rrbracket_{F} \cdot \mathbf{n},\left\{\left\{\mathbf{u}_{h} \cdot \mathbf{v}_{h}\right\}\right\}_{F}\right)_{F} \\
& -\frac{\rho^{f}}{2}\left(\mathbf{z}_{h} \cdot \mathbf{n}, \mathbf{u}_{h} \cdot \mathbf{v}_{h}\right)_{\Sigma} .
\end{aligned}
$$

- The term

$$
\frac{\rho^{f}}{2}\left(\mathbf{u}_{h} \cdot \mathbf{n}, \dot{\mathbf{d}}_{h} \cdot \mathbf{v}_{h}\right)_{\Sigma}
$$

to maintain the consistency of the formulation lost by introducing the fourth term in $c_{h}\left(\mathbf{u}_{h}, \mathbf{u}_{h}, \mathbf{v}_{h}\right)$. We notice that this term guarantees consistency, but probably at the expense of stability. Indeed, with this new term, the relation $(c+c h)\left(\mathbf{u}_{h}, \mathbf{u}_{h}, \mathbf{u}_{h}\right)=0$ does not provide a discrete energy estimate for (5). How could the term (4) be controlled is still an open issue and it is under investigation.

- A stabilizing term $s_{h}$ applied on $\mathcal{F}_{h, \text { com }}^{i}$ to handle spurious pressure and velocity instabilities due to equal order finite elements and to dominating convection regimes, respectively. For example, in this work we considered the continuous interior penalty stabilization (see [12]), as done in [37]. This strategy is characterized by the choice of three parameters, namely, $\gamma_{p}$ in the pressure stabilization term, $\gamma_{\beta}$ in the velocity stabilizaiton term, and $\gamma_{\text {div }}$ in a further term added to give additional control of the incompressibility condition.

- A ghost-penalty term (see [9]), applied on $\mathcal{F}_{h, \Sigma}^{i}$ to guarantee robustness of the method w.r.t. the cut elements, defined as

$$
g_{h}\left(\mathbf{u}_{h}, \mathbf{v}_{h}\right)=\gamma_{g} \sum_{i=1,2} \sum_{F \in \mathcal{F}_{h, \Sigma}^{i}} \mu^{f} h_{F} \int_{F} \llbracket \nabla \mathbf{u}_{h} \rrbracket_{F} \mathbf{n} \cdot \llbracket \nabla \mathbf{v}_{h} \rrbracket_{F} \mathbf{n}
$$

with $\gamma_{g}>0$.

- The terms involving $(\cdot, \cdot)_{\Sigma}$ and $(\cdot, \cdot)_{F}$ that allow one to impose weakly (i.e., in a DG manner) the continuity conditions at the interface $\Sigma$ and at the faces in $\mathcal{F}_{h, p h y}^{i}$, by mimicking the (symmetric) interior penalty method, introduced, for example, in $[18,2]$ for the Poisson problem.

Notice that in problem (3) we have made the choice $\alpha=1 / 2$ for the fluid/fluid mortaring. This is perfectly justified by the homogeneous coupling; see, e.g., [13]. Instead, this choice is not optimal for the fluid/solid mortaring as highlighted in [11]. For this reason, as suggested in [11], we have considered $\alpha=1$ in the numerical experiments reported below.

2.1.3. Full discretization. We denote by $\Delta t>0$ the temporal discretization step and by $(0, T]$ the temporal domain such that $t^{n}=n \Delta t$ for $n=1, \ldots, N_{t}$ with $N_{t}=\frac{T}{\Delta t}$. To ease the presentation, we consider only the implicit Euler scheme for the 
time discretization. The space-time discretization of the problem given by (1) reads as follows: for $n=0, \ldots, N_{t}-1$, find $\left(\mathbf{u}_{h}^{n+1}, p_{h}^{n+1}, \mathbf{d}_{h}^{n+1}\right) \in \mathbf{V}_{h} \times Q_{h} \times \mathbf{W}_{h}$ such that (5)

$$
\left\{\begin{array}{l}
\frac{\rho^{f}}{\Delta t}\left(\mathbf{u}_{h}^{n+1}, \mathbf{v}_{h}\right)_{\Omega^{f}}+a^{f}\left(\mathbf{u}_{h}^{n+1}, \mathbf{v}_{h}\right)+b\left(p_{h}^{n+1}, \mathbf{v}_{h}\right)-b\left(q_{h}, \mathbf{u}_{h}^{n+1}\right)+c\left(\mathbf{u}_{h}^{n}, \mathbf{u}_{h}^{n+1}, \mathbf{v}_{h}\right) \\
+\frac{\rho^{s}}{\Delta t^{2}}\left(\mathbf{d}_{h}^{n+1}, \mathbf{w}_{h}\right)_{\Omega^{s}}+a^{s}\left(\mathbf{d}_{h}^{n+1}, \mathbf{w}_{h}\right) \\
+c_{h}\left(\mathbf{u}_{h}^{n}, \mathbf{u}_{h}^{n+1}, \mathbf{v}_{h}\right)+\frac{\rho^{f}}{2}\left(\mathbf{u}_{h}^{n} \cdot \mathbf{n}, \frac{\mathbf{d}_{h}^{n+1}}{\Delta t} \cdot \mathbf{v}_{h}\right)_{\Sigma}+s_{h}\left(\mathbf{u}_{h}^{n+1}, p_{h}^{n+1} ; \mathbf{v}_{h}, q_{h}\right)+g_{h}\left(\mathbf{u}_{h}^{n+1}, \mathbf{v}_{h}\right) \\
-\left(\alpha \mathbf{T}^{f}\left(\mathbf{u}_{h}^{n+1}, p_{h}^{n+1}\right) \mathbf{n}^{f}+(1-\alpha) \mathbf{T}^{s}\left(\mathbf{d}_{h}^{n+1}\right) \mathbf{n}^{f}, \mathbf{v}_{h}-\mathbf{w}_{h}\right)_{\Sigma} \\
-\left(\mathbf{u}_{h}^{n+1}-\frac{\mathbf{d}_{h}^{n+1}}{\Delta t}, \alpha \mathbf{T}^{f}\left(\mathbf{v}_{h},-q_{h}\right) \mathbf{n}^{f}+(1-\alpha) \mathbf{T}^{s}\left(\mathbf{w}_{h}\right) \mathbf{n}^{f}\right)_{\Sigma} \\
+\frac{\gamma_{\Sigma} \mu^{f}}{h}\left(\mathbf{u}_{h}^{n+1}-\frac{\mathbf{d}_{h}^{n+1}}{\Delta t}, \mathbf{v}_{h}-\mathbf{w}_{h}\right)_{\Sigma} \\
-\sum_{i=1,2} \sum_{F \in \mathcal{F}_{h, p h y}^{i}}\left(\left\{\left\{\mathbf{T}^{f}\left(\mathbf{u}_{h}^{n+1}, p_{h}^{n+1}\right)\right\}\right\}_{F} \mathbf{n}^{f}, \llbracket \mathbf{v}_{h} \rrbracket_{F}\right)_{F} \\
-\sum_{i=1,2} \sum_{F \in \mathcal{F}_{h, p h y}^{i}}\left(\llbracket \mathbf{u}_{h}^{n+1} \rrbracket_{F},\left\{\left\{\mathbf{T}^{f}\left(\mathbf{v}_{h},-q_{h}\right)\right\}_{F} \mathbf{n}^{f}\right)_{F}\right. \\
+\sum_{i=1,2} \sum_{F \in \mathcal{F}_{h, p h y}^{i}} \frac{\gamma_{p h y}^{v} \mu^{f}}{h_{F}}\left(\llbracket \mathbf{u}_{h}^{n+1} \rrbracket_{F}, \llbracket \mathbf{v}_{h} \rrbracket_{F}\right)_{F} \\
=\frac{\rho^{f}}{\Delta t}\left(\mathbf{u}_{h}^{n}, \mathbf{v}_{h}\right)_{\Omega^{f}}+\frac{2 \rho^{s}}{\Delta t^{2}}\left(\mathbf{d}_{h}^{n}, \mathbf{w}_{h}\right)_{\Omega^{s}}+\frac{\rho^{s}}{\Delta t^{2}}\left(\mathbf{d}_{h}^{n-1}, \mathbf{w}_{h}\right)_{\Omega^{s}}+\frac{\rho^{f}}{2}\left(\mathbf{u}_{h}^{n} \cdot \mathbf{n}, \frac{\mathbf{d}_{h}^{n}}{\Delta t} \cdot \mathbf{v}_{h}\right)_{\Sigma} \\
+\left(\frac{\mathbf{d}_{h}^{n}}{\Delta t}, \alpha \mathbf{T}^{f}\left(\mathbf{v}_{h},-q_{h}\right) \mathbf{n}^{f}+(1-\alpha) \mathbf{T}^{s}\left(\mathbf{w}_{h}\right) \mathbf{n}^{f}\right)_{\Sigma}-\frac{\gamma_{\Sigma} \mu^{f}}{h}\left(\frac{\mathbf{d}_{h}^{n}}{\Delta t}, \mathbf{v}_{h}-\mathbf{w}_{h}\right)_{\Sigma}
\end{array}\right.
$$

$\forall\left(\mathbf{v}_{h}, q_{h}, \mathbf{w}_{h}\right) \in \mathbf{V}_{h} \times Q_{h} \times \mathbf{W}_{h}$, and where we have used a first order extrapolation to treat the nonlinearity of the convective term.

The algebraic linear system associated with equation (5) reads

$$
R \mathbf{U}=\mathbf{F},
$$

where

$$
\begin{gathered}
R=\left[\begin{array}{ccc}
K^{f}+C\left(\mathbf{U}^{n}\right)+G+E^{\mathbf{u u}}+H^{\mathbf{u u}} & B^{T}+E^{\mathbf{u} p}+H^{\mathbf{u} p} & E^{\mathbf{u d}} \\
-B+E^{\mathbf{u} p T}+H^{\mathbf{u} p} & S & E^{\mathbf{d p}} \\
E^{\mathbf{u d}} \mathbf{d}^{T} & E^{\mathbf{d} \mathbf{p}^{T}} & K^{s}+E^{\mathbf{d d}}
\end{array}\right], \\
\mathbf{U}=\left[\begin{array}{c}
\mathbf{U}^{n+1} \\
\mathbf{P}^{n+1} \\
\mathbf{D}^{n+1}
\end{array}\right] \text { and } \quad \mathbf{F}=\left[\begin{array}{c}
\mathbf{F}_{\mathbf{u}} \\
\mathbf{F}_{p} \\
\mathbf{F}_{\mathbf{d}}
\end{array}\right] .
\end{gathered}
$$

We have set $K^{f}=\Delta t^{-1} M^{f}+A^{f}$ and $K^{s}=\Delta t^{-2} M^{s}+A^{s}$, where $M^{f}, A^{f}, B$, and $C\left(\mathbf{U}^{n}\right)$ represent the standard matrices of the finite element discretization of the Navier-Stokes problem, $M^{s}$ and $A^{s}$ the mass and stiffness matrices related to the structure discretization, $S$ the matrix related to the fluid stabilization, and $G$ the matrix associated with the ghost penalty term. The matrices $E$ contain the DG terms that couple the fluid and the structure on the interface $\Sigma$, while the matrices $H$ contain the DG terms that ensure the weak continuity of the velocity and stresses on the faces $\mathcal{F}_{h, p h y}^{i}$. 
2.2. The case of geometrically moving structure. In this section, we extend the unfitted formulation presented in the previous section to the case of a geometrically moving structure.

First, we have to introduce the time dependence into the definitions of the domains. Now, the fluid domain is indicated by $\Omega^{f}(t)$, the structure domain is indicated by $\Omega^{s}(t)$, and the fluid-structure interface is defined as $\Sigma(t)=\bar{\Omega}^{f}(t) \cap \bar{\Omega}^{s}(t)$. To ease the presentation, we assume that $\Gamma^{f}=\partial \Omega^{f}(t) \backslash \Sigma(t)$ and $\Gamma^{s}=\partial \Omega^{s}(t) \backslash \Sigma(t)$ are fixed in time. This implies that the domain $\Omega=\bar{\Omega}^{f}(t) \cup \bar{\Omega}^{s}(t)$ is fixed in time and that only the FS interface is moving.

Since the structure problem is solved in a Lagrangian framework, we need to introduce the reference configuration of the solid domain, which will be indicated

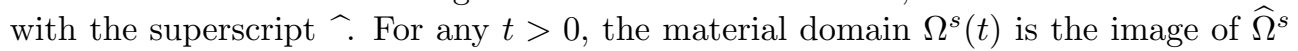
by a Lagrangian map $\mathcal{L}(t): \widehat{\Omega}^{s} \rightarrow \Omega^{s}(t)$. We use the notation $\widehat{g}=g \circ \mathcal{L}(t)$ to denote in $\widehat{\Omega}^{s}$ any function $g$ defined in the current solid configuration $\Omega^{s}(t)$.

The fluid-structure interaction problem reads as follows: find for each $t \in(0, T]$, the fluid velocity $\mathbf{u}$, the fluid pressure $p$, and the solid displacement $\mathbf{d}$, such that

$$
\begin{array}{lr}
\rho^{f} \partial_{t} \mathbf{u}+\rho^{f} \mathbf{u} \cdot \nabla \mathbf{u}-\nabla \cdot \mathbf{T}^{f}(\mathbf{u}, p)=\mathbf{0} & \text { in } \Omega^{f}(t), \\
\nabla \cdot \mathbf{u}=0 & \text { in } \Omega^{f}(t), \\
\mathbf{u}=\mathbf{0} & \text { on } \Gamma^{f} ; \\
\rho^{s} \partial_{t t} \widehat{\mathbf{d}}-\nabla \cdot \widehat{\mathbf{T}}^{s}(\widehat{\mathbf{d}})=\mathbf{0} & \text { in } \widehat{\Omega}^{s}, \\
\widehat{\mathbf{d}}=\mathbf{0} & \text { on } \Gamma^{s} ; \\
\mathbf{u}=\dot{\mathbf{d}} & \text { on } \Sigma(t), \\
\mathbf{T}^{f}(\mathbf{u}, p) \mathbf{n}^{f}=-\mathbf{T}^{s}(\mathbf{d}) \mathbf{n}^{s} & \text { on } \Sigma(t),
\end{array}
$$

where we have used the following formula to pass from the Piola-Kirchhoff tensor $\widehat{\mathbf{T}}^{s}(\widehat{\mathbf{d}})$ to the Cauchy stress tensor $\mathbf{T}^{s}(\mathbf{d})$ :

$$
\widehat{\mathbf{T}}^{s}=J \mathbf{T}^{s} \mathbf{F}^{-T} \text {. }
$$

Here, $J=\operatorname{det}(\mathbf{F}), \mathbf{F}=\nabla \mathbf{x}$ is the deformation tensor, the gradient being taken with respect to the reference space coordinates, and $\mathbf{x}$ are the coordinates of points in the current configuration. For the definitions of the parameters and the other quantities, we refer to the discussion presented in section 2.1.1. We point out that the coupling conditions (6f) $-(6 \mathrm{~g})$ are written in the current configuration.

At the discrete level, we now have the major issue given by the fact that the fluid domain is moving due to the movement of the interface. In particular, although the fluid mesh is fixed at the background, the intersections with the structure one are changing in time. It could even happen (as in the simulation we will describe in section 4.3) that the intersected tetrahedra are not the same from a time step to the following one. In this case, the fully discrete formulation is given again by (5), where however we have to account for two major changements:

(i) Treat the geometric nonlinearity given by the fact that we are solving the fluid equations in an Eulerian configuration. Here, we decide to use an explicit treatment, where at time $t^{n}$ the fluid equations are solved in $\Omega_{h}^{f, n-1}=\Omega \backslash\left(\Omega_{h}^{s, n-1}\right)=\Omega \backslash$ $\left(\mathcal{L}_{h}^{n-1}\left(\widehat{\Omega}^{s}\right)\right)$, where $\mathcal{L}_{h}^{n-1}=I_{\widehat{\Omega}^{s}}+\widehat{\mathbf{d}}_{h}^{n-1}$. Thus, the integrals over the fluid domain and the FS interface are intended in $\Omega_{h}^{f, n-1}$ and $\Sigma_{h}^{n-1}$, respectively, and the structure problem is written in the reference configuration. 

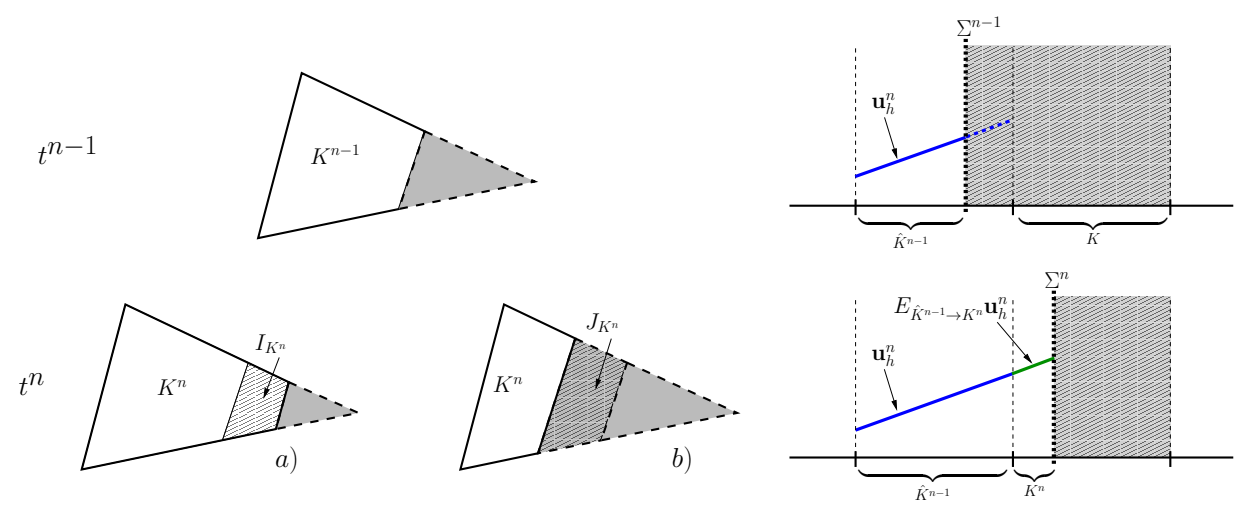

FIG. 6. Left: two possible evolutions of the physical portion of a tetrahedron $K$. (a) the structure moves toward the right, partially uncovering the tetrahedron, so that the corresponding new physical portion $I_{K^{n}}$ is not empty; (b) the structure moves toward the left, increasing the covered region of the tetrahedron, so that the corresponding new covered portion $J_{K^{n}}$ is not empty. Right: example of natural extension operator $E$.

(ii) The fluid velocity at previous time step $\mathbf{u}_{h}^{n-1}$ appearing in the convective term $c_{h}$, in the corresponding consistency term, and in the term coming from time discretization, should be properly defined in the new mesh, since it does not belong to the same space of the test functions $\mathbf{v}_{h}$. In particular, owing to point (i) above, $\mathbf{u}_{h}^{n-1}$ is evaluated on $\Omega_{h}^{f, n-1}$. We have to understand how to write this term on $\Omega_{h}^{f, n}$. To this aim, given two neighboring tetrahedra $K$ and $\widehat{K}$, we indicate by $K^{m}$ and $\widehat{K}^{m}$ their physical (uncovered) portions (polyedra) at the generic time step $t^{m}$, by $I_{K^{n}}=K^{n} \backslash K^{n-1}$ the new physical portion of $K$ uncovered passing from $t^{n-1}$ to $t^{n}$, and by $J_{K^{n}}=K^{n-1} \backslash K^{n}$ the physical portion of $K$ covered passing from $t^{n-1}$ to $t^{n}$; see Figure 6, left.

Then, we first introduce the "natural extension" operator $E_{\widehat{K}^{n-1} \rightarrow K^{n}}$ of a piecewise linear function $w_{h}^{n}$ defined on $\Omega_{h}^{f, n-1}$, which evaluates the linear function $\left.w_{h}^{n}\right|_{\widehat{K}^{n-1}}$ onto $K^{n}$, by extending its values outside $\widehat{K}^{n-1}$; see Figure 6 , right. Thus, we define the following new quantity $\Pi^{n} \mathbf{u}_{h}^{n}$ to be used in (5) in place of $\mathbf{u}_{h}^{n}$ :

$\left.\left(\Pi^{n} \mathbf{u}_{h}^{n}\right)(\mathbf{x})\right|_{K^{n}}= \begin{cases}\left.\mathbf{u}_{h}^{n}(\mathbf{x})\right|_{K^{n-1}} & \text { if } K^{n} \equiv K^{n-1} \text { or }\left(\left|J_{K^{n}}\right|>0 \text { and } \mathbf{x} \in K^{n}\right) \\ E_{K^{n-1} \rightarrow K^{n}} \mathbf{u}_{h}^{n}(\mathbf{x}) & \text { if }\left|I_{K^{n}}\right|>0 \\ \text { not defined } & \text { if }\left|J_{K^{n}}\right|>0 \text { and } \mathbf{x} \in J_{K^{n}} \\ E_{\bar{K}^{n-1} \rightarrow K^{n}} \mathbf{u}_{h}^{n}(\mathbf{x}) & \text { if }\left|K^{n-1}\right|=0 \text { and }\left|K^{n}\right|>0\end{cases}$

where $\bar{K}$ is an arbitrary neighboring tetrahedron with $\left|\bar{K}^{n-1}\right|>0$. The idea is to obtain the numerical solution in a physical polyedron, which was partially covered by the structure at time $t^{n-1}$, by linearly extending the solution available at time $t^{n-1}$ in the same tetrahedron. In the case where $K^{n-1}$ was completely covered by the structure, we linearly extend the numerical solution of a selected neighbor.

For the treatment of the interface position, we refer the reader also to [1] for the membrane case.

3. Implementation details. In this section, we describe the main issues that arise during the implementation of the proposed XFEM/DG approach, in particular 

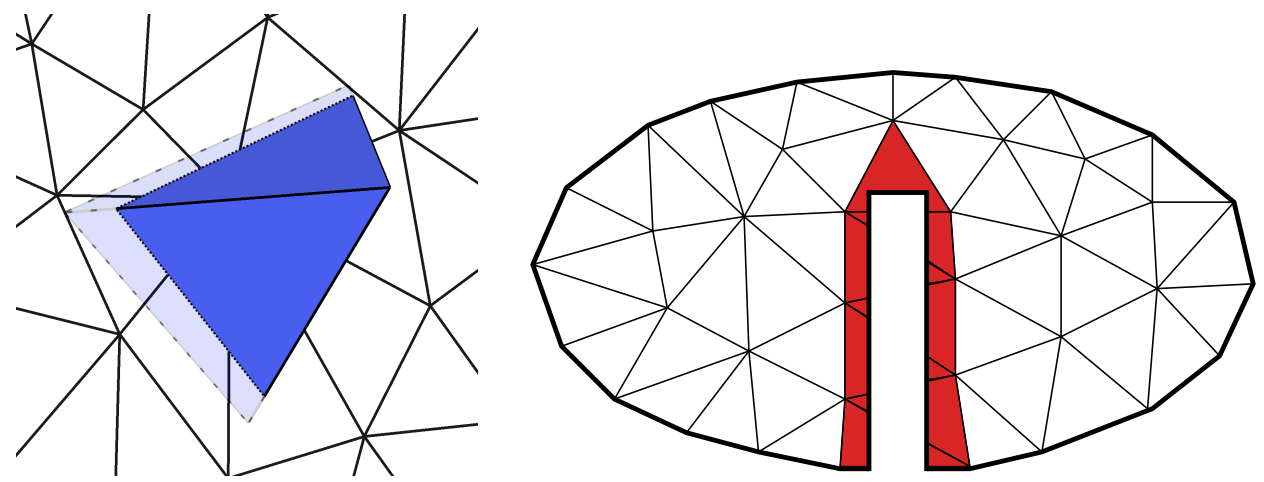

FIG. 7. Left: example of a tetrahedron (blue) of the background mesh that intersects the interface $\Sigma$ (white mesh). The darker part represents the physical part, i.e., the cut-element, the portion of the element that is not overlapped by the foreground mesh, while the lighter part is the overlapped one. Right: the cut-mesh associated with the background mesh shown in Figure 2 that contains cut-elements (in red).

the specific features that characterize the method with respect to the standard finite element method.

In what follows, we indicate with

- Background mesh, the fluid mesh $\mathcal{T}_{h}^{f}$ that covers the entire domain.

- Foreground mesh, the solid mesh $\mathcal{T}_{h}^{s}$ that covers the solid domain and overlaps the fluid one.

- Cut-entities, the physical portion of the geometrical entities (tetrahedra or faces) of the background mesh partially covered by the foreground mesh, which, in the case of volumes or faces, are in general polyhedra or polygon; see Figure 7. In particular, we refer to cut-elements and cut-faces for the case of 3D and two-dimensional (2D) entities, respectively.

- Cut-mesh, the fluid mesh resulting from the difference between the background mesh and the foreground one (see Figure 7, right) that coincides with the physical portion of the background mesh (i.e., the union of cut-entities and elements not covered by the foreground mesh).

Due to the unfitted nature of the meshes, it is necessary to identify which parts of the entities in the background mesh are physical (i.e., belonging to the cut-mesh) with the aim of computing the integrals over these portions. The main steps to address are

1. the computation of the intersections between the unfitted meshes;

2. the generation of the cut-elements and cut-mesh;

3. the addition of the extended dofs according to the XFEM philosophy;

4. the integration over the cut-elements and cut-faces.

In the following sections, we explain how we deal with each of these topics.

3.1. Intersection between meshes. To identify the physical portions of the cut-entities it is necessary to compute the intersection points between the background and foreground mesh. These intersections will be used to reconstruct the cut-elements and the cut-faces on the interface.

Referring to Figure 8, we show two configurations where the intersections points between the background and interface meshes are represented: on the left, we consider the case of a fluid element associated to only one cut-element, while on the right we 

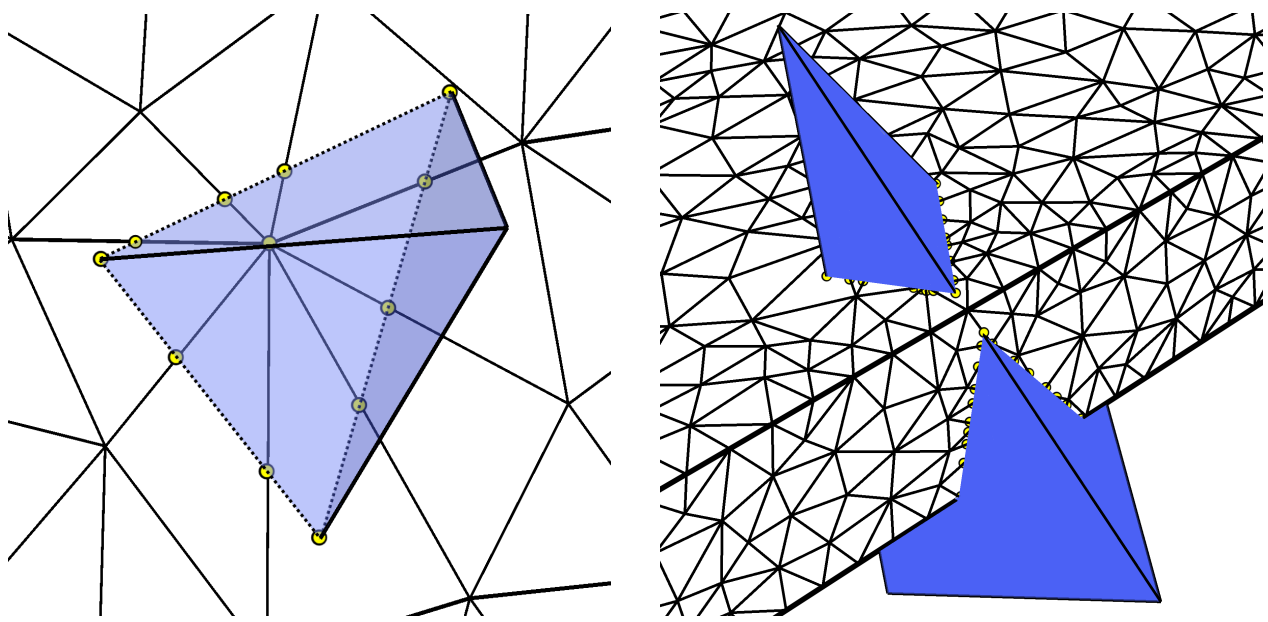

FIG. 8. The foreground mesh (in white) ad the physical part of the tetrahedron (in blue). The resulting intersection points are highlighted (in yellow). Left: one fluid physical element is generated. Right: two fluid physical elements are generated.

consider the case of two cut-elements. The computation of the intersection points may be very expensive from a computational viewpoint, since it is not known a priori which elements of the background mesh are intersected, so a naive procedure may be to check for all the elements of the foreground mesh if they are intersected by an element of the background mesh. To avoid this and to obtain an efficient algorithm, we rely on an alternating digital tree; see $[7,20]$. This data structure, given a bounding box of an entity of the foreground mesh, allows one to check if the bounding box intersects the elements of background mesh and returns a list these elements. Once the list is obtained, we proceed to compute the intersection points. A similar strategy has been already employed, for example, in [30].

At the end of this procedure, for each element of the background mesh that is cut by the foreground one, we have the corresponding list of intersection points.

3.2. Generation of the cut-mesh. The intersection points calculated are stored to generate a subtetrehedralization inside each cut-element, which in general is a polyhedron. The purpose of this tetrahedralization is twofold: (i) integrate over the cut-elements and the cut-faces, and (ii) visualize the numerical solution on the physical part (cut-elements), avoiding the visualization of the solution on the nonphysical portions of the interface background elements. The first point will be explained in detail in section 3.4 .

The subtetrahedralization has to fulfill two requirements: (i) we have to force the intersection points to be vertices of the final tetrahedralization; (ii) the possible additional vertices introduced by the tetrahedralization have to lie inside the element, otherwise the conformity between facing element will be lost. To satisfy these requirements, for each element $K$, we proceed as follows:

1. A one-dimensional (1D) mesh for each edge of $K$ is generated by using the intersection points that lie on the edge as vertices; see Figure 9, left-center.

2. A 2D mesh for each face of $K$ is generated by using the edges computed at step 1 to define the boundary of the face, and by using the intersection points that lie of the face as vertices; see Figure 9, center-right. 

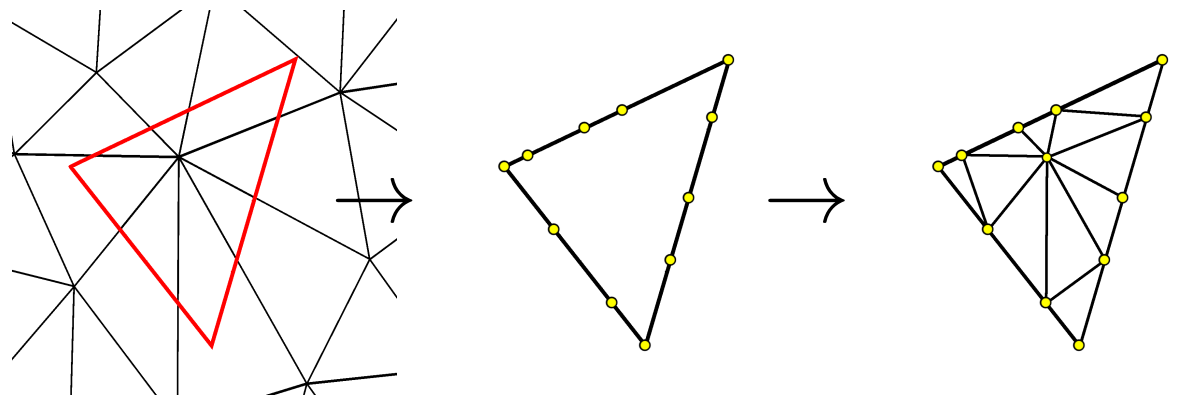

Fig. 9. Subtriangulation of the face of the element in Figure 8. Left: face of the background mesh (in red) and foreground mesh (in white). Center: generation of the $1 D$ meshes over the edges. Right: generation of the $2 D$ mesh over the face. The intersection points are highlighted in yellow.
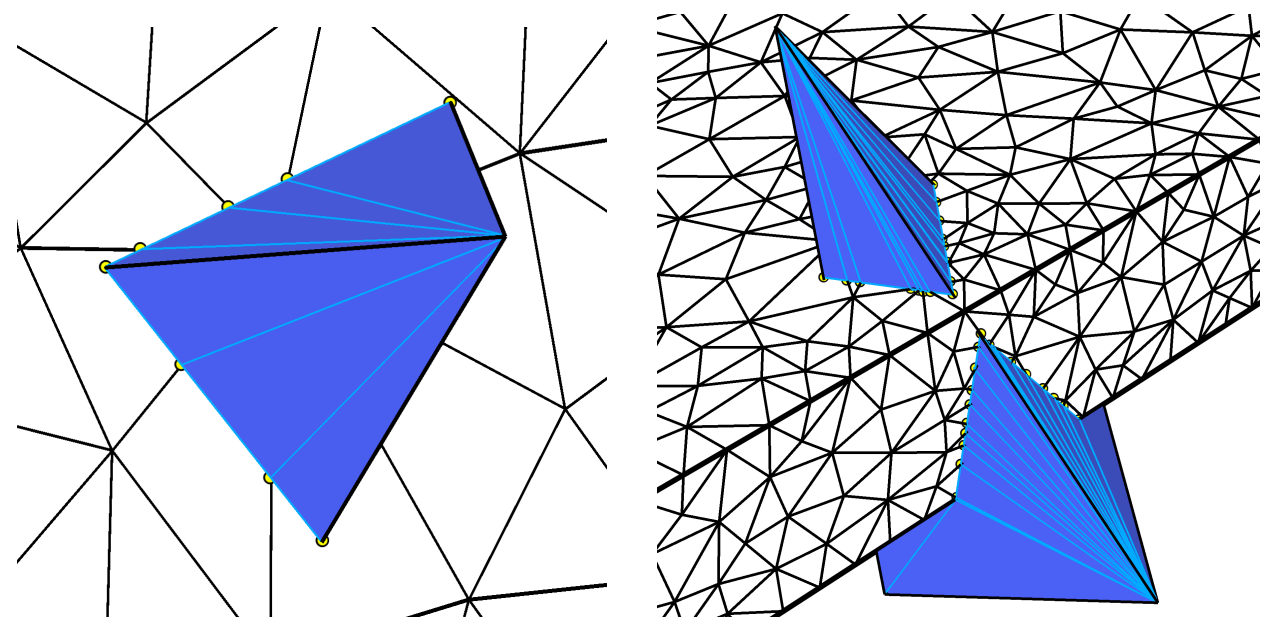

FIG. 10. Tetrahedralization of the element, with the edges of the subtetrahedra highlighted in light blue. Left: element with one fluid physical element. Right: element with two fluid physical elements.

3. A 3D mesh is generated by using the faces computed at step 2 to define the boundary of the element, and by using the intersection points that lie inside the volume of the element.

Steps 2 and 3 are carried out by Triangle [38] and TetGen [39], respectively. In Figure 10, we report the subtetrahedralization for the cases presented in Figure 8.

3.3. Extended and cancelled degrees of freedom. The main goal of the tetrahedralization is the computation of the integrals over the cut-entities. To this aim, it is first necessary to identify which dofs should be selected when integrating an element of the background mesh. In particular, which dofs are cancelled since covered by the foreground mesh, and which dofs are doubled since the corresponding element is subdivided into two or more disconnetted cut-entities. To ease the presentation, in what follows we refer to the dofs associated with conforming piecewise linear finite element.

The elements of the background mesh can be divided into three categories:

(a) Elements that are nonoverlapped by the foreground mesh (see Figure 11, left) or that are partially overlapped by it producing a single connected cut-entity; 

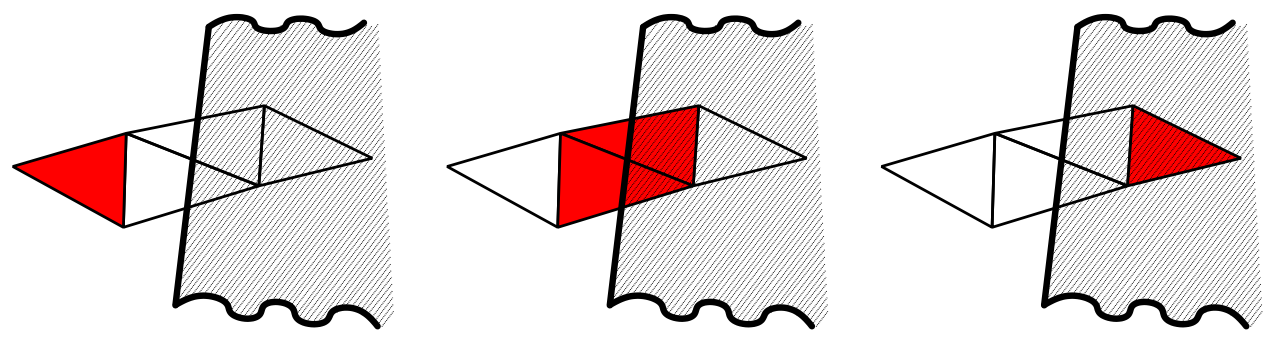

FIG. 11. Possible scenarios of background elements (in red) with respect to the foreground mesh (in grey). From left to right: element not overlapped, two elements partially overlapped, element completely overlapped.
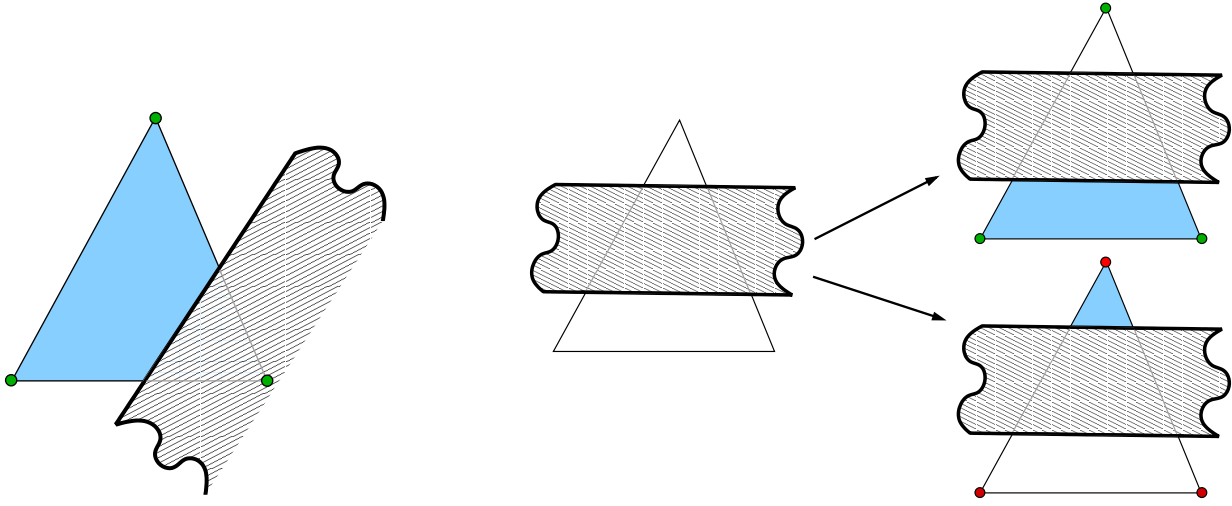

FIG. 12. The two main configurations that may appear in the case of partially overlapped elements. Left: case of one physical element (in blue), the foreground mesh (in grey), and the dofs highlighted in green. Right: case of two physical elements with the background element (in white) and the foreground mesh (in grey). In this case, the set of dofs are doubled (in green and red) and each set is used to compute the solution only in one of the two physical element (in blue).

see Figure 11, center. In this case, we select the dofs of the standard FEM and integration proceeds as usual.

(b) Elements that are partially-overlapped by the foreground mesh, producing two or more disconnetted cut-entities; see Figure 8, right. In this case, we have to consider additional dofs in the spirit of XFEM.

(c) Elements that are fully overlapped by the foreground mesh; see Figure 11, right. In this case, we do not have to consider any dof on the element since the latter does not belong to the physical domain.

In the case (b) above, we have to double the finite element, i.e., the geometric entity and its associated dofs; see Figure 12, right. Hence, we will use a first set of dofs to compute the integrals over one physical element, and the second set of dofs to compute the integrals over the other physical element; see, e.g., [26]. Notice that the case of more than two disconnetted cut-entities corresponding to a partially covered interface background element is treated in an analogous way.

We notice that a similar strategy to handle the FSI problem for unfitted meshes in the case of thick structure has been studied in [11] as well. However, in that paper the authors do not consider that a fluid element could be cut by the solid into two physical parts, and thus they do not need to double the dofs. In some sense our approach is similar to that proposed in [37] even if in that paper the authors consider only a fluid problem, not an FSI one. 

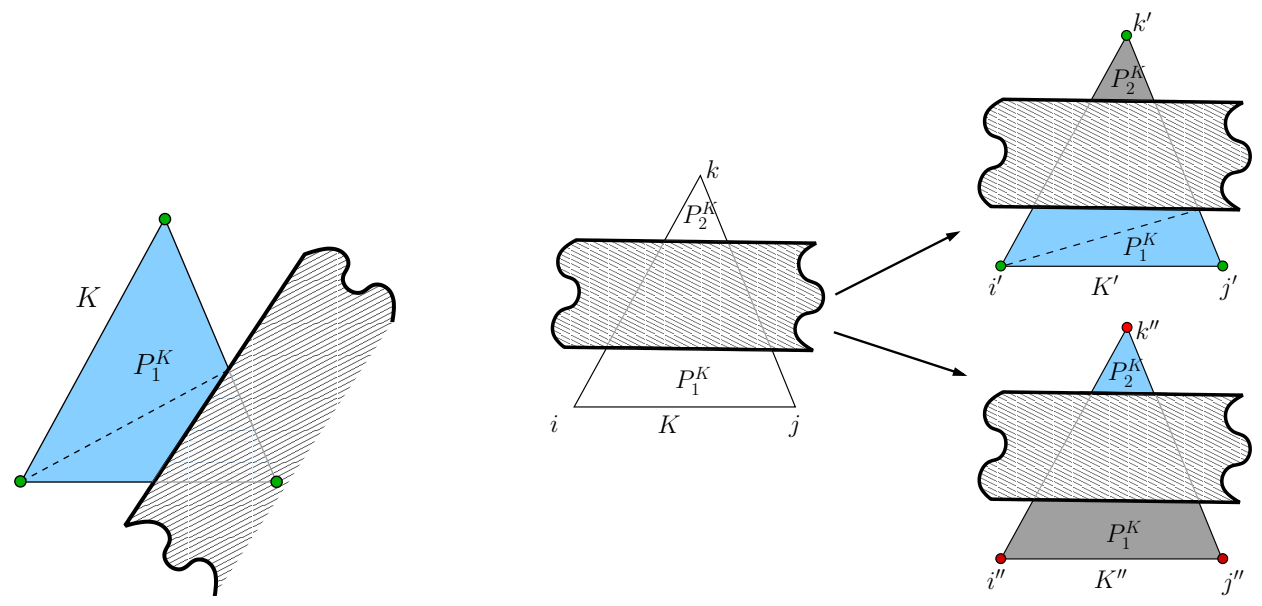

FIG. 13. Computation of the integrals over the partially overlapped elements. Left: case of one physical part (in blue) with the dofs highlighted in green. Right: case of two physical parts (in blue). The integral on each part uses a different set of dofs, $i^{\prime}, j^{\prime}, k^{\prime}$ (in green) for $P_{1}^{K}, i^{\prime \prime}, j^{\prime \prime}, k^{\prime \prime}$ (in red) for $P_{2}^{K}$

3.4. Integration over the cut-entities. The integration over the physical portions of the partially overlapped elements, which, in general, are complex polyhedra, requires one to consider advanced numerical integration techniques. Some of these techniques are presented, e.g., in [33, 32, 41, 44].

To avoid implementing new numerical quadrature formula and to reuse the classical Gaussian quadrature rule available in a standard FEM implementation, we proceed instead as follows: by using the tetrahedralization generated inside each partially overlapped element, we compute the integral over each subtetrahedron and then we sum up all the contributions.

By referring to the configurations shown in Figure 13

- in the case of one physical part (left), we sum the integrals calculated on each subtetrahedron of the polyhedron $P_{1}^{K}$ arisen after the tetrahedralization of the latter by using the dofs defined on the entire element $K$,

- in the case of two physical parts (right), we sum the integrals calculated on each subtetrahedron of $P_{1}^{K}$ by using the dofs indicated by $i^{\prime}, j^{\prime}, k^{\prime}$, and the same strategy is applied on each subtetrahedron of $P_{2}^{K}$ by using the dofs indicated by $i^{\prime \prime}, j^{\prime \prime}, k^{\prime \prime}$.

We point out that the same procedure is applied also when considering the cutfaces for integrating the DG terms on the fluid-structure interface and on the fluid-fluid interface (i.e., on $\Sigma$ and $\mathcal{F}_{h, p h y}^{i}$, respectively; see section 2).

This procedure is able to treat efficiently cases of high geometric complexity and in particular the case of split elements.

4. Numerical examples. In this section, we present some numerical results aiming at assessing the effectiveness of the proposed method. We present three test cases:

I. a linear steady FSI problem (see section 4.1),

II. a nonlinear time-dependent FSI problem with a geometrically fixed structure (see section 4.2),

III. a nonlinear time-dependent FSI problem with a moving structure (section 4.3). 
For each case, we provide a validation of our method, by means of a comparison with the solution obtained with a fitted/conforming finite element method, obtained by means of the ALE formulation [17]. For the latter case, the same values of the stabilization parameters considered for the unfitted case have been used. In particular, for test case I, we compute for different values of $h$, the relative $L^{2}$ norms in space of the structure displacement and fluid pressure differences on a selected line, i.e.,

$$
\operatorname{diff}_{r e l, q}^{l}=\sqrt{\frac{\int_{0}^{L}\left|q^{f i t t}(l)-q^{u n f i t t}(l)\right|^{2} d l}{\int_{0}^{L}\left|q^{f i t t}(l)\right|^{2} d l}},
$$

where $q$ is either the displacement $d$ or the pressure $p, L$ the length of the line, and $l$ the corresponding spatial coordinate. Instead, for test cases II and III, we compute for different values of $h$ or $\Delta t$, the relative $L^{2}$ norms in time of the structure displacement differences in a selected point $\mathbf{p}$, i.e.,

$$
\operatorname{diff}_{r e l, d}^{t}=\sqrt{\frac{\int_{0}^{T}\left|d^{f i t t}(\mathbf{p}, t)-d^{u n f i t t}(\mathbf{p}, t)\right|^{2} d t}{\int_{0}^{T}\left|d^{f i t t}(\mathbf{p}, t)\right|^{2} d t}} .
$$

Notice that for all the cases the structure mesh is the same for the fitted and unfitted cases. We will report the number of tetrahedra and the corresponding mesh size for the unfitted case. Of course, these values change for the fitted background mesh, but since they are almost identical to the unfitted one, we will skip this information later on. All the proposed examples are simulated in a 3D framework with a linear Hooke law for the structure. Moreover, the linear system arising at each time step is solved monolithically with GMRES preconditioned by a $2 \times 2$ block Gauss-Seidel preconditioner, the two blocks being identified by the fluid and structure subproblems, respectively.

The method presented in section 2 and 3 has been implemented in the $\mathrm{C}++$ finite element library LifeV (www.lifev.org).

4.1. Steady and linear FSI problem (test case I). In the following test case, we consider the steady-state solution of a viscous fluid that interacts with a linear elastic thick solid in the small deformations regime. In particular, we consider the Stokes equation for the fluid and the Hooke law for the solid. In this case, the velocity continuity condition at the interface $\Sigma$ is $\mathbf{u}=\mathbf{0}$, and the stresses continuity condition reads $\mathbf{T}^{f} \mathbf{n}^{f}=-\mathbf{T}^{s} \mathbf{n}^{s}$. We obtain the following problem:

$$
\left\{\begin{array}{lr}
-\nabla \cdot\left(-p \mathbf{I}+2 \mu^{f} D(\mathbf{u})\right)=\mathbf{0} & \text { in } \Omega^{f}, \\
\nabla \cdot \mathbf{u}=0 & \text { in } \Omega^{f}, \\
-\nabla \cdot\left(\lambda^{s}(\nabla \cdot \mathbf{d}) \mathbf{I}+2 \mu^{s} D(\mathbf{d})\right)=\mathbf{0} & \text { in } \Omega^{s}, \\
\mathbf{u}=\mathbf{0} & \text { on } \Sigma, \\
\mathbf{T}^{f}(\mathbf{u}, p) \mathbf{n}^{f}=-\mathbf{T}^{s}(\mathbf{d}) \mathbf{n}^{s} & \text { on } \Sigma
\end{array}\right.
$$

with $\mu^{f}=0.035$ poise, $\lambda^{s}=\frac{E \nu}{(1+\nu)(1-2 \nu)}, \mu^{s}=\frac{E}{2(1+\nu)}$, and $E=10^{4} d y n e / \mathrm{cm}^{2}$ is the Young's modulus and $\nu=0.45$ is the Poisson's ratio. We consider the domain $\Omega=(0,1)^{3} \mathrm{~cm}, \Omega^{s}=(0.15,0.85) \mathrm{cm} \times(0.4,0.6) \mathrm{cm} \times(0.31,0.34) \mathrm{cm}$, and $\Omega^{f}=\Omega \backslash \bar{\Omega}^{s}$; see Figure 14. Regarding the boundary conditions, we impose $\mathbf{T}^{f} \mathbf{n}=(0,0,-2) d y n e / \mathrm{cm}^{2}$ on $\Gamma_{\text {in }}, \mathbf{T}^{f} \mathbf{n}=\mathbf{0}$ on $\Gamma_{\text {out }}$ and $\mathbf{u}=\mathbf{0}$ on the remaining fluid boundary, where $\mathbf{n}=$ 


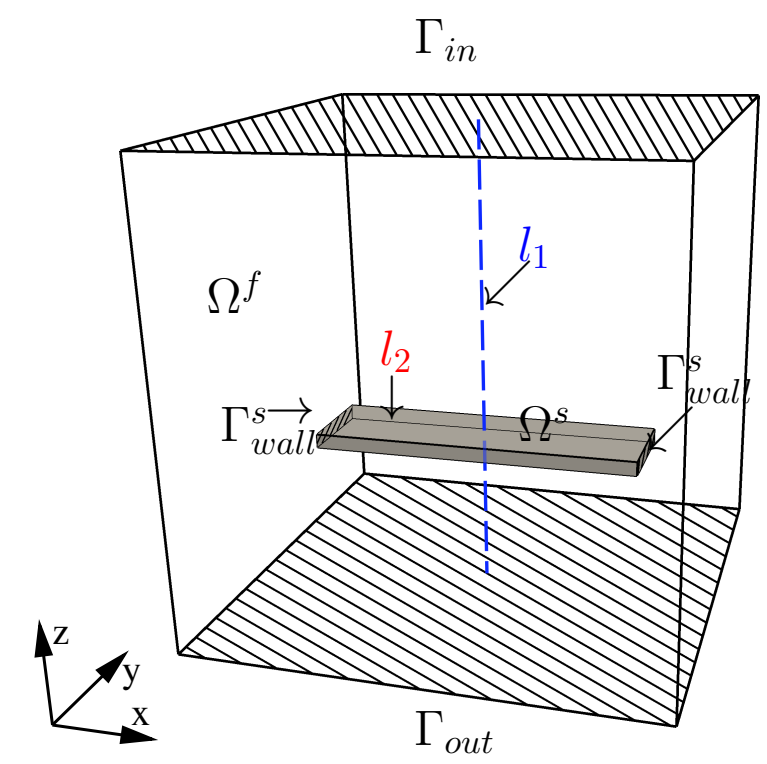

FIG. 14. Sketch of the domain $\Omega$ with the domain $\Omega^{s}$ highlighted in grey. Test case I.

TABLE 1

Number of tetrahedra and corresponding value of h (in brackets) for three levels of refinement, and relative differences for the displacement on the line $l_{2}$ and pressure on the line $l_{1}$. Test case $\mathrm{I}$.

\begin{tabular}{l|c|c|c|c}
\hline & \# tetrahedra in $\mathcal{T}_{h}^{f}$ & \# tetrahedra in $\mathcal{T}_{h}^{s}$ & $\operatorname{diff}_{r e l, d}^{l}$ & $\operatorname{diff}_{r e l, p}^{l}$ \\
\hline Refinement 1 & $75 \mathrm{k}(0.049)$ & $41 \mathrm{k}(0.010)$ & $10.3 \%$ & $3.9 \%$ \\
Refinement 2 & $253 \mathrm{k}(0.035)$ & $114 \mathrm{k}(0.007)$ & $7.0 \%$ & $2.3 \%$ \\
Refinement 3 & $801 \mathrm{k}(0.024)$ & $301 \mathrm{k}(0.005)$ & $5.3 \%$ & $1.6 \%$ \\
\hline
\end{tabular}

$\mathbf{n}^{f}=-\mathbf{n}^{s}$. On the solid we impose $\mathbf{d}=\mathbf{0}$ at $\Gamma_{\text {wall }}^{s}=\{0.15\} \mathrm{cm} \times(0.4,0.6) \mathrm{cm} \times$ $(0.31,0.34) \mathrm{cm} \cup\{0.85\} \mathrm{cm} \times(0.4,0.6) \mathrm{cm} \times(0.31,0.34) \mathrm{cm}$, so that it is fixed on the two sides. We impose the interface conditions (7d) and (7e) on $\Sigma=\partial \Omega^{s} \backslash \Gamma_{\text {wall }}^{s}$.

We choose $\gamma_{\Sigma}=10^{3}, \gamma_{p h y}^{v}=10^{3}, \gamma_{p}=10^{-2}, \gamma_{g}=1, \gamma_{\beta}=\gamma_{d i v}=0$ and we consider three different couples of fluid-structure meshes; see Table 1.

In Figure 15, we report the fluid velocity field and the structure displacement obtained by the XFEM/DG unfitted method for Refinement 3. In Figure 16, top, for the same refinement, we plot the fluid pressure field on the plane $y=0.5 \mathrm{~cm}$ that cuts the structure domain into two parts. From these results, we observe the different value of pressure upstream and downstream the structure. A quantitative representation of the pressure is shown in Figure 16, bottom-left, along the line $l_{1}: x=0.5 \mathrm{~cm}, y=$ $0.5 \mathrm{~cm}, 0 \leq z \leq 1 \mathrm{~cm}$, and of the structure displacement in the $z$-coordinate is shown in Figure 16, bottom-right, along the line $l_{2}: 0.15 \leq x \leq 0.85 \mathrm{~cm}, y=0.5 \mathrm{~cm}, z=$ $0.325 \mathrm{~cm}$; see Figure 14. From these results, we can observe the jump of the pressure field across the structure and the good agreement between the fitted and unfitted solutions. This is also confirmed by the relative differences reported in Table 1 that highlight the increasing agreement between the two solutions for decreasing $h$.

Finally, in Figure 17, we show for Refinement 1 a detail of the pressure field on a slice at $x=0.5 \mathrm{~cm}$. We see that, though some fluid elements are divided by the structure into two unconnected portions, it is possible to approximate a discontinuous solution within the same geometrical element. 

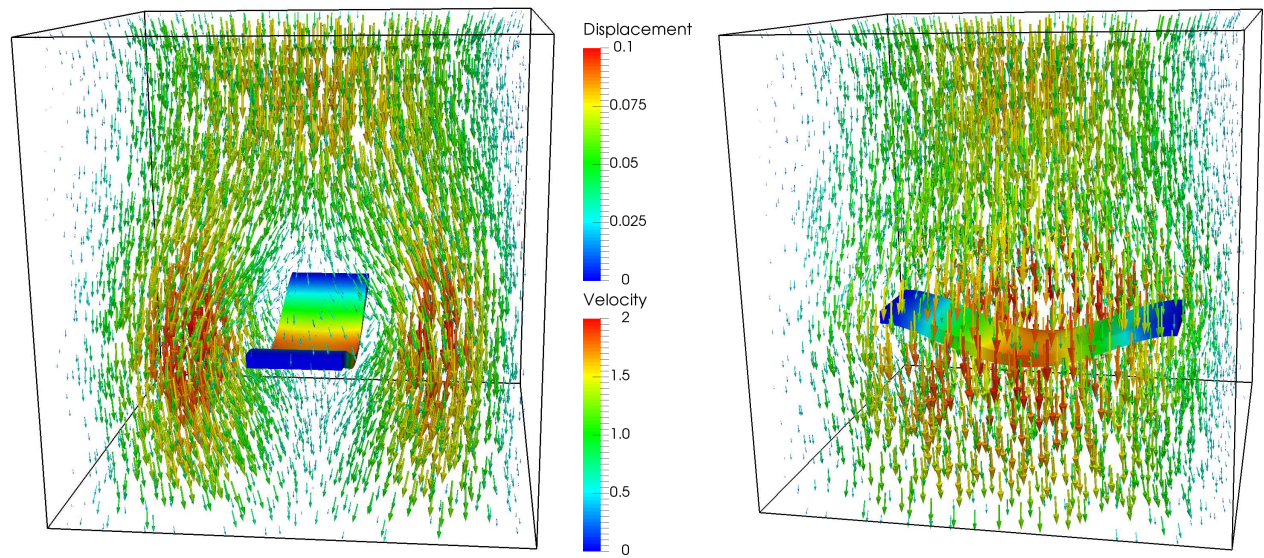

FIG. 15. Plot of the fluid velocity field (in $\mathrm{cm} / \mathrm{s}$ ) and structure displacement magnitude (in $\mathrm{cm})$. Test case I, Refinement 3 .

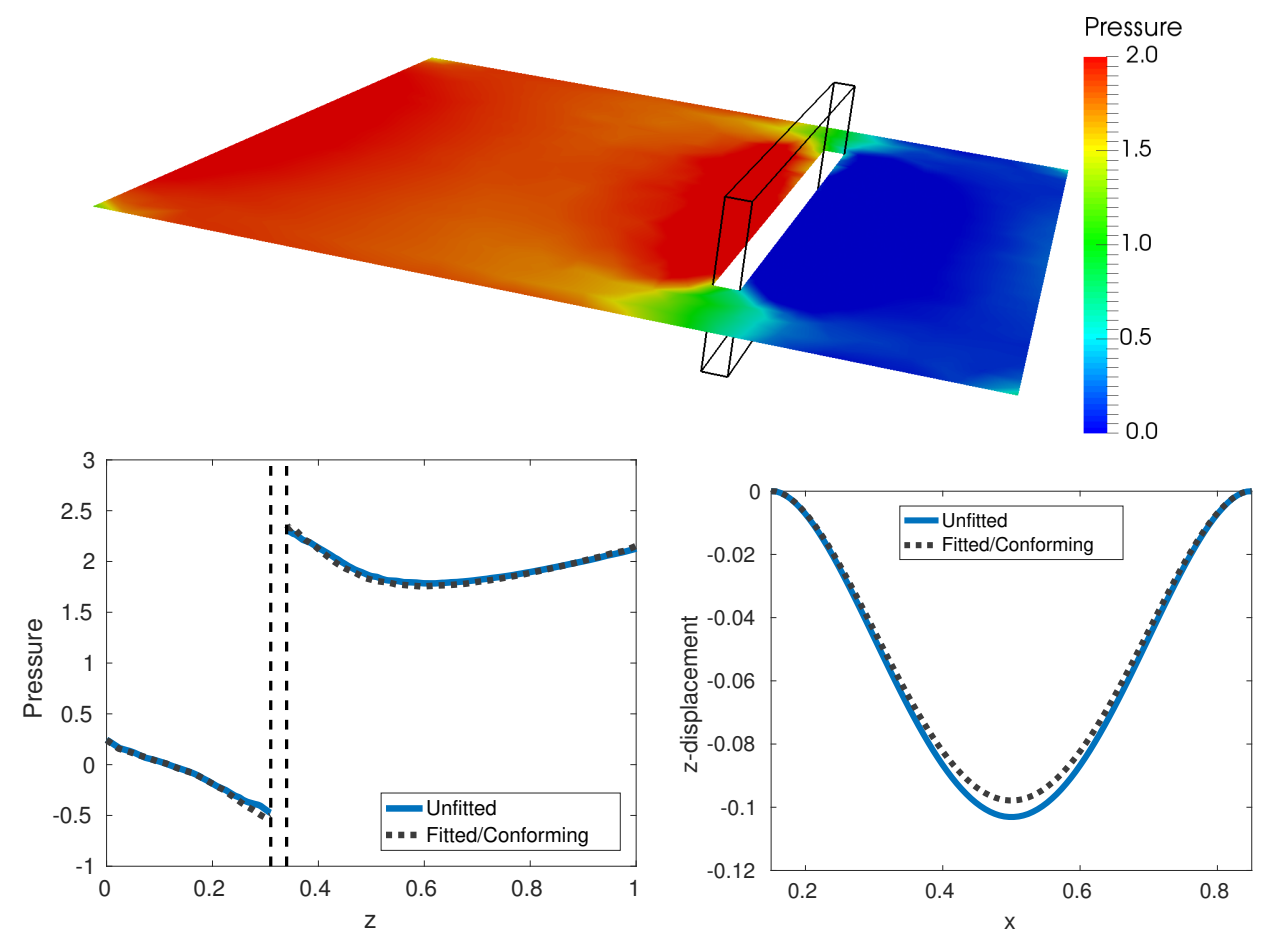

FIG. 16. Top: plot of the fluid pressure field (in dyne $/ \mathrm{cm}^{2}$ ) on a slice located at $y=0.5 \mathrm{~cm}$. The outline of the structure is represented in black. Bottom: plot in the direction of the flow (line $l_{1}$ ) of the fluid pressure (in dyne $/ \mathrm{cm}^{2}$ ) (left) and structure displacement in the $z$-coordinate (in $\mathrm{cm}$ ) (right). The dashed lines at $z=\{0.31,0.34\} \mathrm{cm}$ represent the extremities of the structure. Test case I, Refinement 3.

4.2. Time-dependent nonlinear FSI problem: Geometrically fixed structure (test case II). We consider a time-dependent FSI problem in the small deformations regime given by the coupling of the Navier-Stokes equations for the fluid and the linear elastic Hooke law for the structure. In this case we do not move the interface, but the latter is physically coupled with the fluid. 


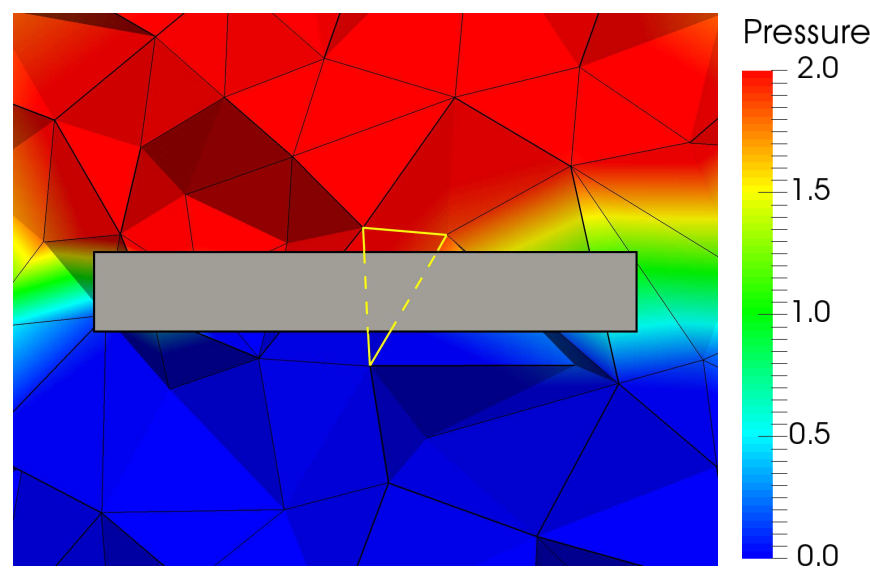

FIG. 17. Detail of the fluid pressure field (in dyne $/ \mathrm{cm}^{2}$ ) near the structure. It is possible to identify some tetrahedra that present a discontinuous solution within the same element, as the one highlighted in yellow. Test case I, Refinement 1.

\section{TABLE 2}

Number of elements and corresponding mesh size (in brackets) for the fluid and solid meshes used for the comparison between the unfitted case and the fitted/conforming finite element method and relative differences for the displacement in the center of mass of the structure. $\Delta t=0.01 \mathrm{~s}$. Test case II.

\begin{tabular}{l|c|c|c}
\hline & \# tetrahedra in $\mathcal{T}_{h}^{f}$ & \# tetrahedra in $\mathcal{T}_{h}^{s}$ & $\operatorname{diff}_{\text {rel,d }}^{t}$ \\
\hline Refinement 1 & $17 \mathrm{k}(0.034)$ & $4 \mathrm{k}(0.015)$ & $7.51 \%$ \\
Refinement 2 & $52 \mathrm{k}(0.023)$ & $13 \mathrm{k}(0.010)$ & $4.40 \%$ \\
Refinement 3 & $134 \mathrm{k}(0.017)$ & $29 \mathrm{k}(0.008)$ & $3.28 \%$ \\
\hline
\end{tabular}

We consider the same domain of test case I, but with different dimensions; in particular we set $\Omega=(0,0.7) \mathrm{cm} \times(0,0.3) \mathrm{cm} \times(0,0.3) \mathrm{cm}, \Omega^{s}=(0.1,0.6) \mathrm{cm} \times$ $(0.1,0.2) \mathrm{cm} \times(0.135,0.165) \mathrm{cm}$, and $\Omega^{f}=\Omega \backslash \bar{\Omega}^{s}$. We impose a zero stress condition, $\mathbf{T}^{f} \mathbf{n}=\mathbf{0}$, at the top and at the bottom of the fluid domain and $\mathbf{u}=\mathbf{0}$ on the lateral walls of the fluid boundary. As in the steady-state case, the solid is kept fixed on two of its extremities. Moreover, we apply the following volumetric force:

$$
\mathbf{f}^{s}(t)= \begin{cases}\rho^{s} \mathbf{k} & \text { if } t \in(0,0.1) s, \\ 0 & \text { if } t \in[0.1, T] s,\end{cases}
$$

where $\mathbf{k}=(0,0,1) \mathrm{cm} / \mathrm{s}^{2}$ and $T=1.5 \mathrm{~s}$. On the fluid-structure interface we impose the continuity of the kinematic and dynamic conditions. As initial conditions, we set $\mathbf{u}(\mathbf{x}, 0)=\mathbf{0}, \mathbf{d}(\mathbf{x}, 0)=\mathbf{0}$ and $\dot{\mathbf{d}}(\mathbf{x}, 0)=\mathbf{0}$. The physical parameters are set as follows: $\rho^{f}=1 \mathrm{~g} / \mathrm{cm}^{3}, \rho^{s}=0.1 \mathrm{~g} / \mathrm{cm}^{3}, \mu^{f}=0.035$ poise, $E=10^{3} \mathrm{dyne} / \mathrm{cm}^{2}, \nu=0.45$. We consider three different couples of fluid-structure meshes; see Table 2 and three values of the time step, namely, $\Delta t=0.02,0.01,0.005 \mathrm{~s}$. Finally, we choose $\gamma_{\Sigma}=$ $10, \gamma_{p h y}^{v}=10^{3}, \gamma_{p}=10^{-1}, \gamma_{\beta}=0.5, \gamma_{\text {div }}=0.5, \gamma_{g}=1$.

In Figure 18, we show the numerical solution at different time steps for Refinement 3. Again, the proposed method is able to capture the structure dynamics. In order to validate these results, we perform a comparison between the presented unfitted formulation and the ALE conforming finite element method. In Figure 19, we report the structure displacements in the $z$-coordinate of the solid center of mass along time 


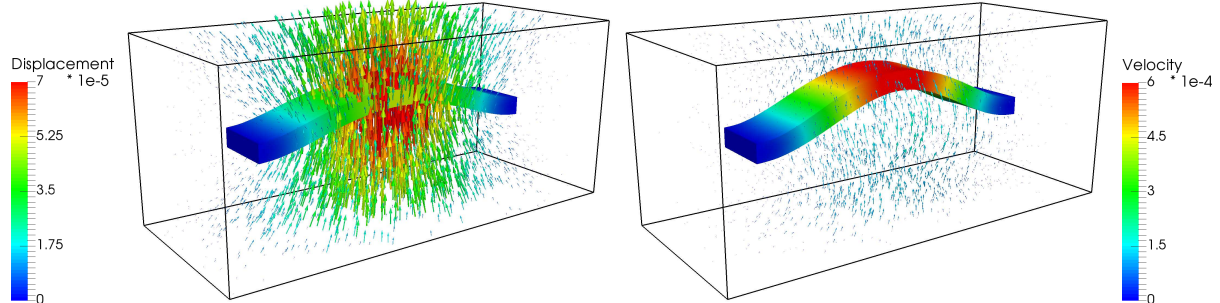

FIG. 18. Numerical solution at different time steps: $t=0.11$ s (left), $t=0.19 \mathrm{~s}$ (right). Fluid velocity field (in $\mathrm{cm} / \mathrm{s}$ ) and structure displacement (in $\mathrm{cm}$ ). Test case II.
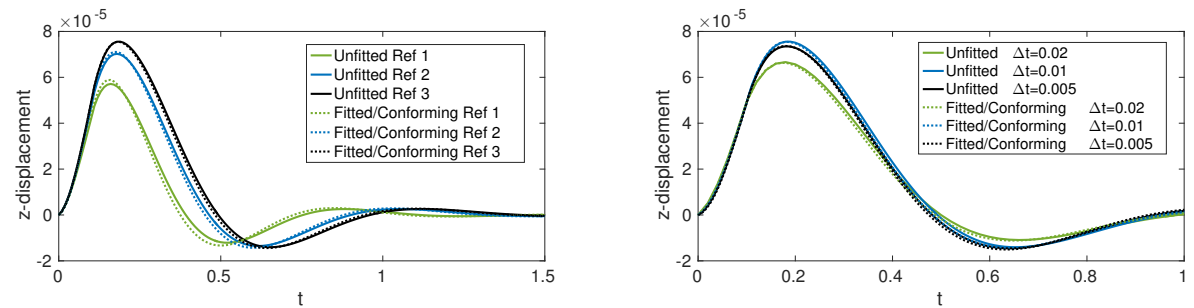

FIG. 19. Structure displacement in the z-coordinate along time of the solid center of mass. Comparison between the XFEM/DG-unfitted and the ALE-conforming methods. Left: space refinement, $\Delta t=0.01$. Right: time refinement, Refinement 3. Test case II.

TABLE 3

Relative differences for the displacement in the center of mass of the structure for different $\Delta t$ for the comparison between the unfitted case and the fitted/conforming FEM. Refinement 3. Test case II.

\begin{tabular}{c|c}
\hline$\Delta t$ & diff $_{r e l, d}^{t}$ \\
\hline 0.02 & $3.72 \%$ \\
0.01 & $3.25 \%$ \\
0.005 & $2.34 \%$ \\
\hline
\end{tabular}

for space and temporal refinements. In Tables 2 and 3, we report the corresponding relative differences for space and temporal refinements, respectively. These results show that the differences reduces by increasing the refinement of the meshes and of $\Delta t$.

4.3. Time-dependent nonlinear FSI problem: Moving structure (test case III). In this section, we show the numerical results obtained in the case of a dynamic structure, which is geometrically moved. We consider a time-dependent FSI problem given by the coupling of the Navier-Stokes equations for the fluid and the linear elastic Hooke law for the structure, as described in section 2.2. We employ the same domains considered in test case II reported in section 4.2. We impose a periodic sine function at the inlet, i.e., $\mathbf{u}=\left(0,0,-2.5 \sin \left(\frac{\pi}{8} t\right)\right) \mathrm{cm} / \mathrm{s}$ on $\Gamma_{\text {in }}, \mathbf{T}^{f} \mathbf{n}=\mathbf{0}$ on the outlet $\Gamma_{\text {out }}$ and $\mathbf{u}=\mathbf{0}$ on the remaining walls of the fluid boundary. As in test case I, the solid is kept fixed on two of its extremities, i.e., $\mathbf{d}=\mathbf{0}$ at $\Gamma_{\text {wall }}^{s}$. As initial conditions, we set $\mathbf{u}(\mathbf{x}, 0)=\mathbf{0}, \mathbf{d}(\mathbf{x}, 0)=\mathbf{0}$ and $\dot{\mathbf{d}}(\mathbf{x}, 0)=\mathbf{0}$. We also use the following values for the parameters: $\rho^{f}=1 \mathrm{~g} / \mathrm{cm}^{3}, \rho^{s}=0.1 \mathrm{~g} / \mathrm{cm}^{3}, \mu^{f}=$ 0.035 poise, $E=2 \cdot 10^{3}$ dyne $/ \mathrm{cm}^{2}, \nu=0.45$. The Reynolds number is $R e \approx 10$. 

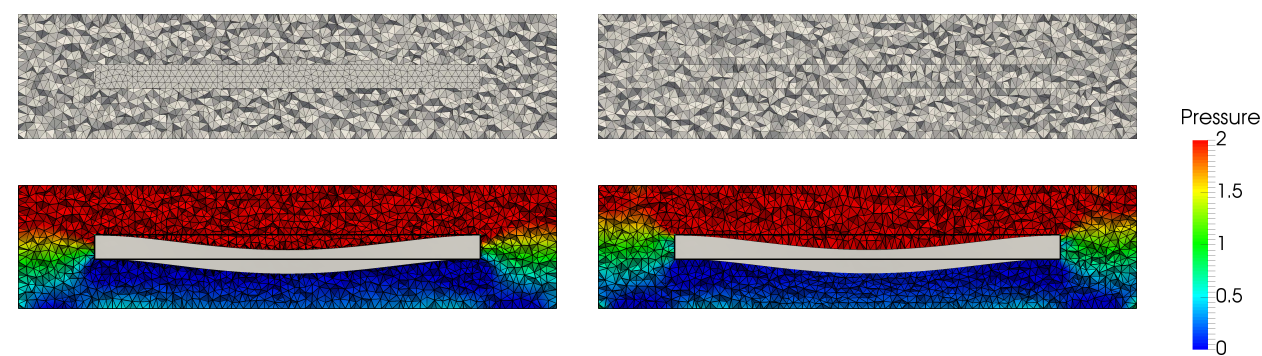

FIG. 20. Up: meshes for XFEM/DG unfitted (left) and ALE/fitted (right) methods. Down: fluid pressure field (in dyne $/ \mathrm{cm}^{2}$ ) and structure location at $t=0.4 \mathrm{~s}$. The moving structure is colored in white, while its initial position is represented by the thick black line, XFEM/DG unfitted (left), ALE/fitted (right). Test case III.

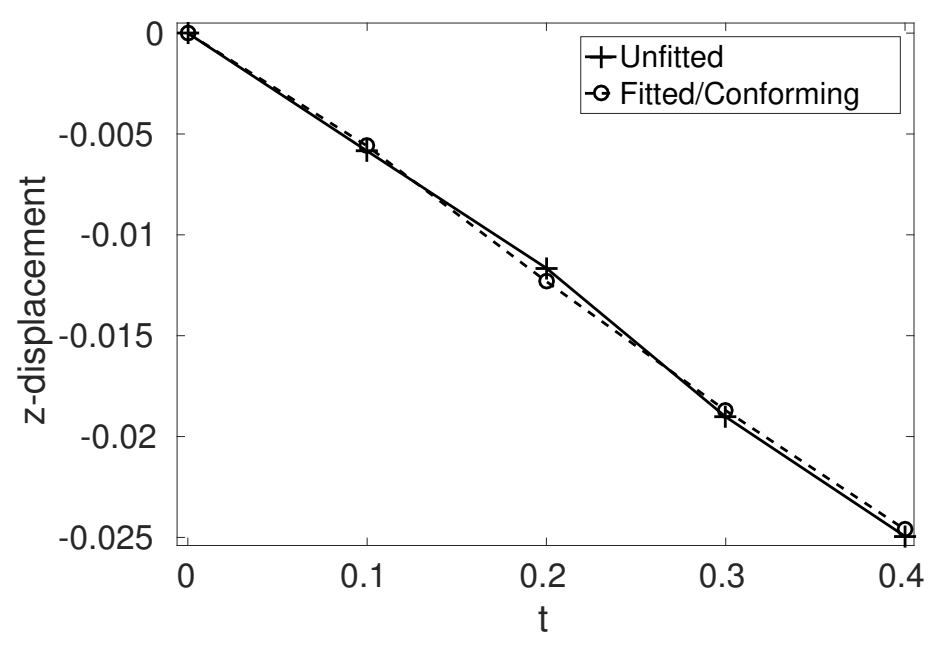

FIG. 21. Displacements of the center of mass of the structure over time obtained with the fitted and unfitted methods. Test case III.

We employ a fluid mesh $\mathcal{T}_{h}^{f}$ composed by $250 k$ tetrahedra and a solid mesh $\mathcal{T}_{h}^{s}$ composed by $14 k$ tetrahedra. The time step $\Delta t$ is $0.1 \mathrm{~s}$. We choose $\gamma_{\Sigma}=10^{2}, \gamma_{\text {phy }}^{v}=$ $10^{3}, \gamma_{p}=10^{-1}, \gamma_{\beta}=0.5, \gamma_{d i v}=0.5$, and $\gamma_{g}=1$.

In Figure 20, we plot for both the XFEM/DG and ALE solutions the pressure field in the fluid domain and represent the moving structure accordingly to the computed displacement at a given time step. In Figure 21, we compare the displacement of the center of mass obtained with the XFEM/DG unfitted and fitted ALE methods. From these results, we observe the excellent agreement between the results obtained with the two methods also in the case of moving interface.

In order to validate the effectiveness of the strategy proposed in section 2.4 to treat the case of physical portions of the background mesh changing in time, we consider a second test with moving interface on a coarser mesh with the same domain as in test case I reported in section 4.1; see Figure 14. We use the same parameters of the previous test case and fluid and structure meshes composed by $38 k$ and $5 k$ tetrahedra, respectively.

In Figure 22, we show the numerical solution on a section of the fluid/solid domain. In particular, we plot again the pressure field in the fluid domain and we represent the moving structure according to the computed displacement at different time steps. 

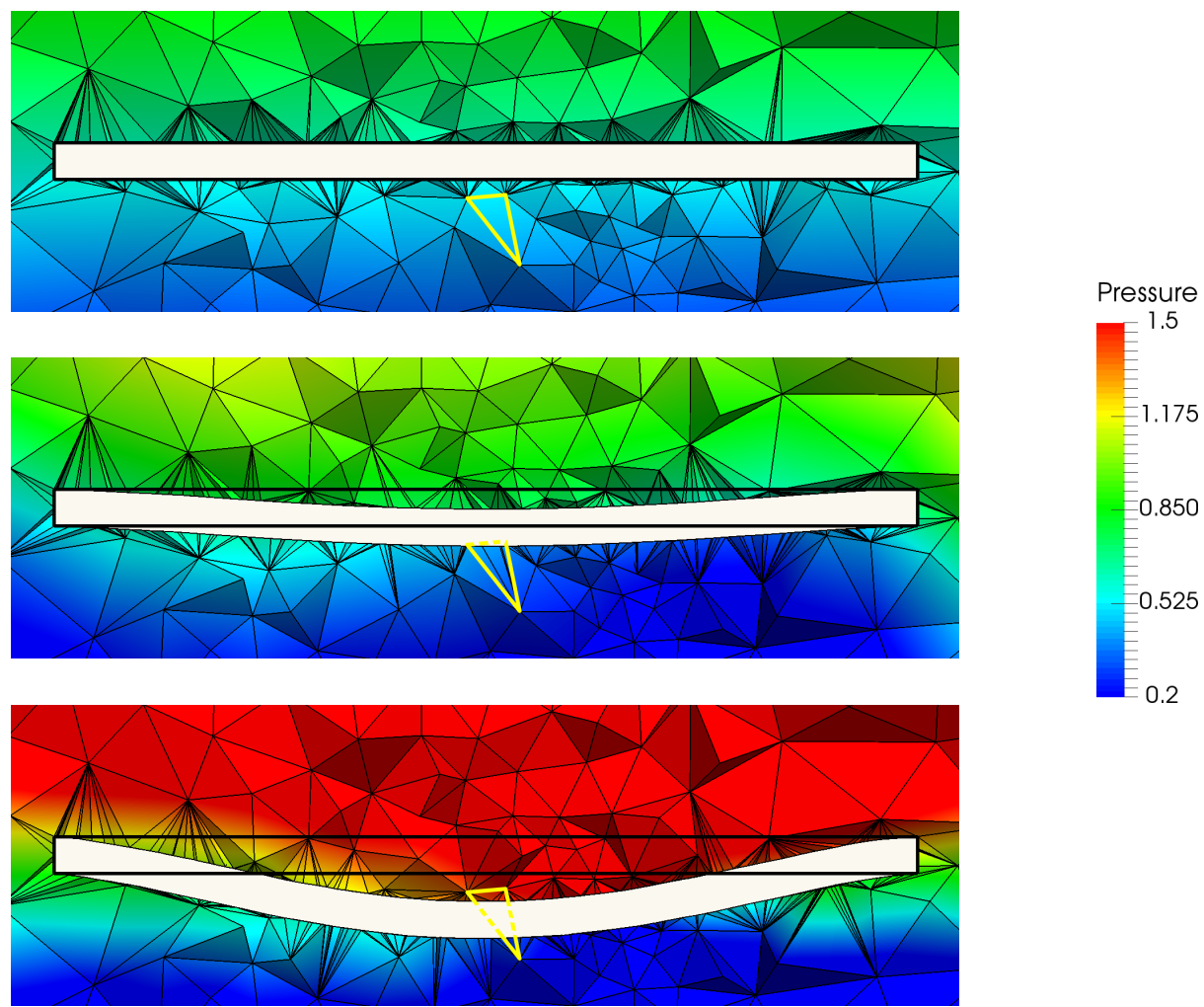

FIG. 22. Fluid pressure field (in dyne $/ \mathrm{cm}^{2}$ ) and structure location at different time steps, from top to bottom: $t=0.1 \mathrm{~s}, t=0.3 \mathrm{~s}$, and $t=0.6 \mathrm{~s}$. The moving structure is colored in white, while its initial position is represented by the thick black line. In yellow, we depict an example of tetrahedron, initially intact, which is cut during the simulation. Test case III.

We see that the fluid elements crossed by the structure change in time as well as the subtetrahedralization computed in such elements.

We notice that in this case, unlike in test case II, the fluid tetrahedra intersected by the structure are changing in time, due to the movement of the structure.

The physical domain follows the movement of the structure without actually moving the fluid mesh, maintaining an accurate description of the solution across the structure and representing the jump in the pressure.

We remark that the procedures to compute the intersections, to generate the cutentities, and to define the dofs need to be performed at each time step. However, the computational cost spent for this processing is very trifling compared to the total time (2.5s vs. $118 s)$.

5. Conclusions and Limitations. In this paper we have considered an unfitted extended finite elements/DG approach for the numerical solution of the fluid-structure interaction problem in the case of a thick structure. To the best of our knowledge, this is the first time that this methodology has been applied to the case of a thick solid. Moreover, this should be the first 3D implementation of XFEM for FSI.

To assess the effectiveness of the proposed method, we reported some $3 \mathrm{D}$ test cases both in the case of a geometrically fixed and a moving structure. Moreover, we provided a first step toward the validation of the presented method, by comparing 
the corresponding results with those obtained with a ALE fitted/conforming finite elements.

The main limitations of this work are the moderate Reynolds numbers of the numerical experiments $(\simeq 10)$ and the simple computational geometries. We are currently working to improve both these points.

This work opened new challenges in the field of unfitted methods to solve FSI problems in $3 \mathrm{D}$, in particular the development of new preconditioners which allow one to speed up convergence and treat greater Reynolds numbers, and the study of efficient techniques which avoid the assembling at each time step of the whole matrices in the linear system.

\section{REFERENCES}

[1] F. Alauzet, B. Fabrèges, M. A. Fernández, and M. Landajuela, Nitsche-XFEM for the coupling of an incompressible fluid with immersed thin-walled structures, Comput. Methods Appl. Mech. Engrg., 301 (2016), pp. 300-335, https://doi.org/10.1016/j.cma.2015.12. 015.

[2] D. N. Arnold, F. Brezzi, B. Cockburn, and L. D. Marini, Unified analysis of discontinuous Galerkin methods for elliptic problems, SIAM J. Numer. Anal., 39 (2001), pp. 1749-1779, https://doi.org/10.1137/S0036142901384162.

[3] M. Astorino, J.-F. Gerbeau, O. Pantz, and K.-F. Traoré, Fluid-structure interaction and multi-body contact: Application to the aortic valves, Comput. Methods Appl. Mech. Engrg., 198 (2009), pp. 3603-3612.

[4] M. Bennoune, J. Morin-Drouin, And R. G. Owens, On the jump conditions for an immersed interface method, SIAM J. Sci. Comput., 38 (2016), pp. A1280-A1316, https://doi.org/10. $1137 / 140989856$.

[5] D. Boffi And L. Gastaldi, A finite element approach for the immersed boundary method, Comput. \& Structures, 81 (2003), pp. 491-501, https://doi.org/10.1016/S0045-7949(02) 00404-2.

[6] D. Boffi, L. Gastaldi, L. Heltai, and C. Peskin, On the hyper-elastic formulation of the immersed boundary method, Comput. Methods Appl. Mech. Engrg., 197 (2008), pp. 22102231.

[7] J. Bonet and J. Peraire, An alternating digital tree (adt) algorithm for $3 D$ geometric searching and intersection problems, Internat. J. Numer. Methods Engrg., 31 (1991), pp. 1-17.

[8] I. BoRAZJANI, Fluid-structure interaction, immersed boundary-finite element method simulations of bio-prosthetic heart valves, Comput. Methods Appl. Mech. Engrg., 257 (2013), pp. 103-116.

[9] E. Burman, Ghost penalty, C. R. Math., 348 (2010), pp. 1217-1220, https://doi.org/10.1016/ j.crma.2010.10.006.

[10] E. Burman And M. Fernández, Stabilized explicit coupling for fluid-structure interaction using Nitsche's method, C. R. Acad. Sci. Paris Sér. I Math., 345 (2007), pp. 467-472.

[11] E. Burman And M. A. Fernández, An unfitted Nitsche method for incompressible fluidstructure interaction using overlapping meshes, Comput. Methods Appl. Mech. Engrg., 279 (2014), pp. 497-514.

[12] E. Burman, M. A. Fernández, and P. Hansbo, Continuous interior penalty finite element method for Oseen's equations, SIAM J. Numer. Anal., 44 (2006), pp. 1248-1274, https: //doi.org/10.1137/040617686.

[13] B. Cockburn, G. Kanschat, And D. Schotzau, A note on discontinuous Galerkin divergencefree solutions of the Navier-Stokes equations, J. Sci. Comput., 31 (2006), pp. 61-73.

[14] S. Court AND M. FourniÉ, A fictitious domain finite element method for simulations of fluidstructure interactions: The Navier-Stokes equations coupled with a moving solid, J. Fluids Struct., 55 (2015), pp. 398-408, https://doi.org/10.1016/j.jfluidstructs.2015.03.013.

[15] J. De Hart, F. BaAijens, G. Peters, and P. Schreurs, A computational fluid-structure interaction analysis of a fiber-reinforced stentless aortic valve, J. Biomech., 36 (2003), pp. 699-712, https://doi.org/10.1016/S0021-9290(02)00448-7.

[16] D. A. Di Pietro And A. ERn, Mathematical Aspects of Discontinuous Galerkin Methods, Math. Appl. 69, Springer, Berlin, 2012.

[17] J. DoneA, An arbitrary Lagrangian-Eulerian finite element method for transient dynamic fluid-structure interaction, Comput. Methods Appl. Mech. Engrg., 33 (1982), pp. 689-723. 
[18] J. Douglas and T. Dupont, Interior Penalty Procedures for Elliptic and Parabolic Galerkin Methods, Springer, Berlin, 1976, pp. 207-216, https://doi.org/10.1007/BFb0120591.

[19] Q. Du, M. D. Gunzburger, L. S. Hou, And J. Lee, Analysis of a linear fluid-structure interaction problem, Discrete Contin. Dyn. Syst., 9 (2003), pp. 633-650.

[20] L. Formaggia, Data structures for unstructured mesh generation, in Handbook of Grid Generation, CRC Press, Boca Raton, FL, 1998.

[21] T.-P. Fries, Overview and comparison of different variants of the XFEM, Proc. Appl. Math. Mech., 14 (2014), pp. 27-30, https://doi.org/10.1002/pamm.201410008.

[22] R. Glowinski, T.-W. Pan, T. I. Hesla, and D. D. JosePh, A distributed Lagrange multiplier/fictitious domain method for particulate flows, Int. J. Multiph. Flow, 25 (1999), pp. 755-794, https://doi.org/10.1016/S0301-9322(98)00048-2.

[23] B. Griffith, X. Luo, D. McQueen, And C. Peskin, Simulating the fluid dynamics of natural and prosthetic heart valves using the immersed boundary method, Int. J. Appl. Mech., 1 (2009), pp. 137-176.

[24] B. E. GRIFFITH, Immersed boundary model of aortic heart valve dynamics with physiological driving and loading conditions, Internat. J. Numer. Methods Biomed. Eng., 28 (2012), pp. $317-345$.

[25] S. HAeri AND J. S. Shrimpton, On the application of immersed boundary, fictitious domain and body-conformal mesh methods to many particle multiphase flows, Int. J. Multiph. Flow, 40 (2012), pp. 38-55.

[26] A. Hansbo and P. Hansbo, An unfitted finite element method, based on nitsches method, for elliptic interface problems, Comput. Methods Appl. Mech. Engrg., 191 (2002), pp. 55375552, https://doi.org/10.1016/S0045-7825(02)00524-8.

[27] Y. G. Lai, K. B. Chandran, and J. Lemmon, A numerical simulation of mechanical heart valve closure fluid dynamics, J. Biomech., 35 (2002), pp. 881-892.

[28] G. Marom, Numerical methods for fluid-structure interaction models of aortic valves, Arch. Comput. Methods Eng., 22 (2015), pp. 595-620, https://doi.org/10.1007/ s11831-014-9133-9.

[29] G. Marom, H.-S. Kim, M. Rosenfeld, E. RaAnani, and R. Haj-Ali, Fully coupled fluidstructure interaction model of congenital bicuspid aortic valves: Effect of asymmetry on hemodynamics., Med. Biol. Eng. Comput., 51 (2013), pp. 839-848, https://doi.org/10. 1007/s11517-013-1055-4.

[30] A. Massing, M. G. Larson, And A. LogG, Efficient implementation of finite element methods on nonmatching and overlapping meshes in three dimensions, SIAM J. Sci. Comput., 35 (2013), pp. C23-C47.

[31] R. Mittal and G. Iaccarino, Immersed boundary methods, Annu. Rev. Fluid Mech., 37 (2005), pp. 239-261, https://doi.org/10.1146/annurev.fluid.37.061903.175743.

[32] S. E. Mousavi And N. Sukumar, Numerical integration of polynomials and discontinuous functions on irregular convex polygons and polyhedrons, Comput. Mech., 47 (2011), pp. 535554, https://doi.org/10.1007/s00466-010-0562-5.

[33] S. Natarajan, D. R. Mahapatra, And S. P. A. Bordas, Integrating strong and weak discontinuities without integration subcells and example applications in an XFEM/GFEM framework, Internat. J. Numer. Methods Engrg., 83 (2010), pp. 269-294, https://doi.org/ 10.1002/nme.2798.

[34] C. Peskin, Flow patterns around heart valves: A numerical method, J. Comput. Phys., 10 (1972), pp. 252-271.

[35] C. Peskin, The immersed boundary method, Acta Numer., 11 (2002), pp. 479-517, https: //doi.org/10.1017/S0962492902000077.

[36] T. RÜBERG AND J. M. G. AzNAR, Numerical simulation of solid deformation driven by creeping flow using an immersed finite element method, Adv. Model. Simul. Eng. Sci., 3 (2016), https://doi.org/10.1186/s40323-016-0061-0.

[37] B. Schott And W. A. Wall, A new face-oriented stabilized xfem approach for $2 d$ and $3 d$ incompressible Navier-Stokes equations, Comput. Methods Appl. Mech. Engrg., 276 (2014), pp. 233-265, https://doi.org/10.1016/j.cma.2014.02.014.

[38] J. R. Shewchuk, Delaunay refinement algorithms for triangular mesh generation, Comput. Geom., 22 (2002), pp. 21-74, https://doi.org/10.1016/S0925-7721(01)00047-5.

[39] H. Si, Tetgen: A Quality Tetrahedral Mesh Generator and Three-Dimensional Delaunay Triangulator, Technical report, WIAS, 2006; also available online from http://wias-berlin.de/ software/tetgen/.

[40] F. Sturla, E. Votta, M. Stevanella, C. A. Conti, And A. Redaelli, Impact of modeling fluid-structure interaction in the computational analysis of aortic root biomechanics, Med. Eng. Phys., 35 (2013), pp. 1721-1730.

Copyright $@$ by SIAM. Unauthorized reproduction of this article is prohibited. 
[41] Y. SUdhaKAR AND W. A. WALL, Quadrature schemes for arbitrary convex/concave volumes and integration of weak form in enriched partition of unity methods, Comput. Methods Appl. Mech. Engrg., 258 (2013), pp. 39-54, https://doi.org/10.1016/j.cma.2013.01.007.

[42] R. Temam, Navier-Stokes Equations, AMS, Providence, RI, 1977.

[43] R. van Loon, P. D. Anderson, F. N. van de Vosse, and S. J. Sherwin, Comparison of various fluid-structure interaction methods for deformable bodies, Comput. \& Struct., 85 (2007), pp. 833-843, https://doi.org/10.1016/j.compstruc.2007.01.010.

[44] G. Ventura and E. Benvenuti, Equivalent polynomials for quadrature in heaviside function enriched elements, Internat. J. Numer. Methods Engrg., 102 (2015), pp. 688-710.

[45] E. Votta, T. B. Le, M. Stevanella, F. Fusini, E. G. Caiani, A. Redaelli, and F. Sotiropoulos, Toward patient-specific simulations of cardiac valves: State-of-theart and future directions, J. Biomech., 46 (2013), pp. 217-228, https://doi.org/10.1016/j. jbiomech.2012.10.026.

[46] E. J. Weinberg and M. R. KaAzempur Mofrad, Transient, three-dimensional, multiscale simulations of the human aortic valve, Cardiovascular Eng., 7 (2007), pp. 140-155, https: //doi.org/10.1007/s10558-007-9038-4.

Copyright (c) by SIAM. Unauthorized reproduction of this article is prohibited. 